\title{
DO SIMILAR COMMUNITIES DEVELOP IN SIMILAR SITES? A TEST WITH ZOOPLANKTON STRUCTURE AND FUNCTION
}

\author{
David G. Jenkins ${ }^{1}$ And Arthur L. Buikema, Jr. \\ Department of Biology, Virginia Polytechnic Institute and State University, Blacksburg, Virginia 24061 USA
}

\begin{abstract}
McCune and Allen (1985) asked the question "Will similar forests develop on similar sites?" and concluded that dissimilar old-growth forests had developed on similar sites due to historical factors (colonization, disturbance, etc.). We asked "Do similar zooplankton communities develop in similar ponds?" We compared zooplankton community structure and function in 12 newly constructed experimental ponds during $1 \mathrm{yr}$ of natural colonization and analyzed a suite of physical-chemical variables to evaluate the assumption of environmental similarity among ponds.

Ponds were similar for the measured environmental variables. However, zooplankton communities were structurally different, as indicated by analyses of species presence/absence, colonization and species accrual curves, and taxa (rotifer, copepod, cladoceran, and Chaoborus) density and biomass. Species varied widely in their colonization abilities. Zooplankton communities also differed in productivity of some taxa and community-level respiration rates.

Scale was important in detecting structure and function differences among zooplankton communities. Species- and taxa-level analyses showed clear differences among communities, but community-level analyses of structure (species richness, total density and biomass) and function (productivity, respiration, and ammonia regeneration rates) could not identify distinct sets of communities. Community structure and function may be comparable in sensitivity for detecting change but need to be compared at equivalent scales.

Dispersal (as evidenced by colonization history) was a regulator of new zooplankton communities, because it did not occur rapidly or uniformly among similar ponds. All zooplankton do not disperse readily. The extent to which dispersal limits older zooplankton communities is unknown, but genetic studies indicate low dispersal rates among established populations. Dispersal also regulates assemblages of organisms expected to be less vagile than zooplankton and in various ecosystems, indicating that "supply-side" and metapopulation concepts are valuable for community ecology.

Priority effects may have lasting influence on subsequent community structure, depending on colonization rates and sequences. We propose explicit recognition (and careful examination) of a commonly assumed but rarely tested "quorum effect": local abiotic and biotic processes regulate communities and arrival processes do not, because potential members have already arrived. Given either priority or quorum effects, dispersal may be an important, often-overlooked process regulating community structure and function, especially when it is not rapid.
\end{abstract}

Key words: colonization history; community composition and assembly; community structure; dispersal; experimental ponds; quorum and priority effects; rotifers; scale effects; zooplankton.

\section{INTRODUCTION}

Do similar communities develop in similar sites? McCune and Allen (1985) asked this question about established, old-growth forests, and concluded that historical factors during early succession had been important in forming dissimilar forests on similar sites. This question is fundamental to understanding the relative importance of stochastic, regional-scale processes vs. deterministic local-scale processes that affect community composition and succession (Ricklefs 1987,

Manuscript received 4 April 1997; revised 18 July 1997; accepted 23 July 1997.

${ }^{1}$ Present address: Biology Department, University of Illinois at Springfield, Springfield, Illinois 62794 USA.
Drake 1991, Caley and Schluter 1997). The historical factors of dispersal, colonization sequence and rate, and priority effects on subsequent community structure, have been shown to be important in various communities: marine fouling (Sutherland and Karlson 1977); coral reef fish (Sale 1977); rocky intertidal (Roughgarden et al. 1987); pond amphibians (Alford and Wilbur 1985); old-growth forests (McCune and Allen 1985); tropical rain forests (Hubbell and Foster 1986); and prairie grasslands (Tilman 1997).

Robinson and Edgemon (1988) experimentally manipulated colonization rate, order, and timing of 28 phytoplankton species into 54 similar laboratory microcosms. All three variables had significant effects on species richness, with colonization rate and timing ac- 
counting for the greatest variation on any given date. Colonization order had a greater influence on species relative abundance when colonization rates were low.

Colonization is a subject bearing a rich history of ecological study, but most of it is related to speciesarea hypotheses and island biogeography. Four species-area hypotheses exist. Equilibrium: species richness results from a balance of immigration and extinction (MacArthur and Wilson, 1967). Random Placement: species richness is due to a random sampling phenomenon, with larger areas simply serving as larger "nets" (Arrhenius 1921, Coleman 1981). Habitat Diversity: larger areas are likely to have more diverse habitats, and therefore support greater species richness (Williams 1943). Intermediate Disturbance: disturbance regime determines species richness and is related to area (Connell 1978). Of the four hypotheses, the Equilibrium hypothesis is most commonly discussed.

Species-area hypotheses attempt to explain species richness on islands or sites of varying size and distances. Consequently, few colonization studies have examined details of community structure, such as contingencies of colonization order or interspecific variation in colonization rates. Also, we know of no studies that have examined the potential effects of colonization dynamics on community function (productivity, respiration, nutrient regeneration) in similar sites. In addition, perceived ecological pattern is scale dependent: community-level metrics such as species richness may mask more detailed patterns (species distribution and timing, density, biomass) that could be considered important. Finally, species-area studies logically involve different-sized sites. Natural colonization patterns in multiple, similar sites have not been adequately studied, despite the potential such studies provide to address some important questions about processes regulating community assembly.

Communities of rapid colonizers should assemble (reach a "quorum") quickly. Thereafter, local processes (e.g., resource limitations and interspecific interactions) should primarily regulate community structure, rather than regional processes (dispersal) and residual effects of colonization history (priority effects). Indeed, this would seem to be the working hypothesis in zooplankton ecology. Zooplankton (especially rotifers) have been commonly considered to be cosmopolitan and to disperse readily by aerial or phoretic transport of cysts, resting eggs, ephippia, or diapaused organisms (e.g., King 1980, Brown and Gibson 1983, Wetzel 1983, Pennak 1989, Begon et al. 1990, Lampert and Sommer 1997). In addition, resource constraints, predation, and competition are often considered to be important regulators of zooplankton communities (e.g., Lampert 1985, Kerfoot and Sih 1987, Carpenter 1988, MacIsaac and Gilbert 1991). To our knowledge, Drake (1991) is the only study of zooplankton colonization dynamics as a regulator of community assembly: he used five cladoceran species, a copepod, an amphipod, and an ostracod in laboratory microcosm experiments for up to $180 \mathrm{~d}$.

The working hypothesis above would also suggest that zooplankton populations in isolated ponds exchange propagules as highly interactive components of regional metapopulations (Gotelli 1991), and should exhibit relatively high rates of gene flow among ponds. However, genetic analyses of some copepod and cladoceran populations indicate otherwise (Boileau and Hebert 1991, Berg and Garton 1994, Hebert and Wilson 1994). Therefore, it would seem appropriate to evaluate zooplankton colonization dynamics as a potential regulator of community structure and function.

Purpose and caveats. - We asked the question "Do similar zooplankton communities develop in similar ponds?" We defined zooplankton communities as being composed of rotifers, copepods, cladocerans, ostracods, and Chaoborus. More specifically, we addressed three questions: (1) Do similarly constructed ponds have similar environmental conditions? (2) If so, do structurally similar zooplankton communities develop in those ponds? (3) And do those zooplankton communities function (i.e., process energy and nutrients) similarly?

In addition, we compared patterns in community structure and function (i.e., productivity, respiration, nutrient regeneration rates) to evaluate potential relationships between the two perspectives of an ecological community (O'Neill et al. 1986). By "function" we do not refer to perceived trophic levels or trophic interactions, but to the energetic and nutrient processing properties of zooplankton assemblages, at the same scales of those traditionally used in studies of colonization (species richness) or energetics (e.g., net primary productivity per unit volume).

We compared a suite of physical-chemical variables and zooplankton community structure and function among 12 new experimental ponds for $1 \mathrm{yr}$. Sample collection and analysis techniques were similar among ponds and over time, and we used the same statistics for each of the three data sets (environment, structure, and function) to facilitate comparisons among the data sets.

The purpose of physical-chemical analyses was to carefully evaluate the assumption of environmental similarity among ponds. Our goal was not to identify direct relationships between environmental variables and zooplankton community dynamics. To do so would require experimental treatments of a greater range than expected among similar ponds.

This study was a "natural experiment" and therefore essentially descriptive. We did not experimentally treat ponds; the only manipulation of the ponds was the addition of well water to compensate for evaporation and/or leaks. We used univariate statistics, but we emphasized multivariate techniques, which are designed to summarize and display patterns (Digby and Kempton 1987). 
Zooplankton generation times are brief (days to months), and zooplankton communities typically undergo seasonal succession (Hutchinson 1967). We expected (and observed) temporal variation in our results, but this paper focuses on comparisons among ponds and selected variables to address our questions above. Also, we considered $1 \mathrm{yr}$ sufficient to examine colonization and population dynamics of multiple zooplankton species over multiple life history cycles (Connell and Sousa 1983). Of course, we cannot consider interannual variation in colonization dynamics and seasonal conditions from our results. This would be best accomplished by starting sets of ponds annually, so that 1 -yr-old ponds could be compared to new ponds, etc. We do hypothesize on the potential importance of our results for subsequent zooplankton communities in the ponds.

\section{Materials ANd Methods}

\section{Study site}

Twelve, identical experimental ponds ( 0.04 ha, maximum depth $=2.1 \mathrm{~m}$ ) were constructed during 1987 1988 at the Southern Piedmont Agricultural Experiment Station near Blackstone, Virginia (see Jenkins 1990, 1995 for additional details). All 12 ponds were filled simultaneously with chlorinated tap water (source: Nottoway Reservoir) over the course of $6 \mathrm{~d}$, ending on 31 January 1988. At no time was water transferred from one pond to another during this study. Water levels were maintained during the remainder of the study with well water. In addition, no fish were present in the ponds during the study.

\section{Sampling and data collection}

Sampling and analyses were conducted biweekly for 1 yr (5 February 1988-10 February 1989), except for one 3-wk interval in November 1988. Additional sampling was conducted during intervening weeks from March through October. Each pond was sampled at three stations from a boat, always at the center (2.14 $\mathrm{m}$ depth) and at two of the four sides (1.07 $\mathrm{m}$ depth, chosen by coin toss). Three types of samples were collected at each station: unfiltered water (for environmental variables and rotifers), preserved net zooplankton (for structure and productivity), and live net zooplankton (for respiration and nutrient regeneration). Station samples were pooled to provide one sample of each type for each pond, per Hurlbert's (1984) recommendations regarding pseudoreplication. Sampling gear was rinsed in $70 \%$ ethyl alcohol or filtered water to avoid dispersing zooplankton among ponds during sampling. However, ponds were sampled with the same boat, which was dragged ashore during sample processing $(\sim 0.5 \mathrm{~h})$ before entering the next pond. Therefore, we sampled ponds in random order as an added precaution against investigator-generated pattern among ponds.
Environmental variables.-Dissolved oxygen (D.O.) and temperature were recorded in each pond at $1 \mathrm{~m}$ with a Yellow Springs Instrument (Yellow Springs, Ohio) Model 54 meter. Secchi depth was measured at the center station. Water was collected at each station with an integrated water column sampler $(1.8 \mathrm{~m}$ long, $5 \mathrm{~cm}$ internal diameter). Five samples were collected at each station, to $1.8 \mathrm{~m}$ depth at the center and $0.9 \mathrm{~m}$ depth at side stations. Two 20-L buckets were filled from each pond and combined. Duplicate subsamples were filtered through Whatman GF/C filters at $\leq 26.7$ $\mathrm{kPa}$ negative pressure with a hand pump (Tarapchak et al. 1982). Filters were stored in sealed test tubes on dry ice until return to the laboratory for chlorophyll $a$ analyses. Filtrate was poured into an acid-washed, borosilicate glass bottle and stored on ice until laboratory analyses for water chemistry (within 5 d). One such bottle was prepared for each pond at each sampling date. Concurrent with this procedure, $\mathrm{pH}$ was measured on an unfiltered subsample with an Orion (Beverly, Massachusetts) Model 407A meter.

Analyses conducted in the laboratory were: chlorophyll $a$, hardness, alkalinity, conductivity, $\mathrm{NH}_{3}-\mathrm{N}$, $\mathrm{NO}_{3}-\mathrm{N}, \mathrm{NO}_{2}-\mathrm{N}$ and soluble reactive $\mathrm{PO}_{4}-\mathrm{P}$ (SRP). All analyses were conducted according to APHA et al. (1985). Chlorophyll $a$ was measured with a PerkinElmer (Norwalk, Connecticut) Model 552 spectrophotometer. Hardness and total alkalinity were measured by titration. Conductivity was measured with a YSI Model 32 conductance meter: all values were standardized to $25^{\circ} \mathrm{C}$. Ammonia-nitrogen and SRP were measured with a Gilford (Corning, New York) Response spectrophotometer, and $\mathrm{NO}_{3}-\mathrm{N}$ and $\mathrm{NO}_{2}-\mathrm{N}$ were measured with a Dionex (Sunnyvale, California) Model 14 ion chromatograph.

Zooplankton community structure.-Crustaceans and Chaoborus larvae were collected with an unmetered Wisconsin-style plankton net $(80-\mu \mathrm{m}$ mesh). The net was gently placed on the bottom at each station and allowed to remain in place for $2 \mathrm{~min}$ before it was hauled up. Chaoborus may be underrepresented in analyses, because they can burrow in sediments during daytime, but this bias should apply equally to all ponds. Two net samples were collected at each station, and all net samples were pooled to provide one sample per pond (Hurlbert 1984). Zooplankton were preserved in $4 \%$ formalin, and buffered with 5\% sodium acetate and 5\% sugar (Steedman 1976, Haney and Hall 1973). Pond depth was recorded at each sampling event and used to calculate water volume collected for estimates of net zooplankton densities.

Rotifers were collected by sieving $4.0 \mathrm{~L}$ of the combined water sample (see Environmental variables above) through $35-\mu \mathrm{m}$ mesh plankton netting (Likens and Gilbert 1970) to provide one sample per pond (Hurlbert 1984). Rotifers were preserved with $4 \%$ buffered formalin as above, separately from net plankton samples. 
Entire rotifer and net zooplankton samples were counted if sparse, while at least three subsamples were counted if a sample was dense, following quantitative dilution. Subsamples were obtained by mixing a sample and removing $1.0 \mathrm{~mL}$ using a calibrated autopipette with a tip cut to a 4-mm opening (Edmondson and Winberg 1971). All Chaoborus larvae were counted. All organisms were identified to species, except a few cases for which species-level identification could not be confirmed.

Individual dry masses were calculated for zooplankton species using literature masses or size-mass formulae (Doohan and Rainbow 1971, Dumont et al. 1975, Bottrell et al. 1976, Ruttner-Kolisko 1977, Makarewicz and Likens 1979, Pace and Orcutt 1981, McCauley 1984). All species were measured with a Filar (Rochester, New York) digital micrometer mounted on the microscope and calibrated with a stage micrometer. A headwidth-dry mass regression was calculated for Chaoborus punctipennis in the ponds, following procedures of McCauley (1984), with the following result: ln dry mass $=5.0325+1.9797$ (ln head width) $($ dry mass in milligrams; head width in millimeters); $R=$ $0.88, N=26$. Rotifer individual dry mass estimates assumed specific gravity $=1.05 \mathrm{~g} / \mathrm{mL}$ and dry mass $=0.15$ (wet mass).

Rotifer dry mass estimates were based on average size per species, so population biomass estimates were based on average size $\times$ population density (Rigler and Downing 1984). All counted crustacean and Chaoborus individuals were measured and individual dry mass estimates were computed from size-mass regressions, so population biomass estimates were based on the sum of individual biomass values (Rigler and Downing 1984).

Zooplankton community structure data were analyzed for colonization patterns per species and pond, colonization curves (species richness per unit time), and species accrual curves (cumulative species richness). Also, population density and biomass data were summarized for taxa (rotifers, copepods, cladocerans, Chaoborus, and ostracods). Density and biomass data for major genera are available in Jenkins (1990). Finally, taxa values were summarized to community-level variables (species richness, density, biomass) for comparisons to community-level function data.

Zooplankton community productivity.-Zooplankton taxa (rotifer, cladoceran, copepod, Chaoborus) productivity and total community productivity were calculated as the sum of appropriate species productivity estimates. Crustacean and Chaoborus productivity were estimated by increment-summation (Morin and Dumont 1994) and dry mass estimates listed in Jenkins (1990). The increment summation method assumes that all individuals within a size class are growing at the same rate. Rigler and Downing (1984) demonstrated the identity of the increment summation and instantaneous growth methods when there is no mortality, but stressed the importance of using the equation appropriate to the organism's growth when mortality is significant. Because we could not collect mortality or growth rate data for all species in all 12 ponds, productivity estimates may be in error if mortality was high and/or if growth rates were exponential. However, productivity was calculated identically for all ponds. Whatever error may be inherent in productivity estimates should be equally applicable for all ponds, and comparisons of ponds should remain valid.

Rotifer productivity was also estimated by increment-summation, using the modification for rotifers described by Rigler and Downing (1984). This method assumes rotifer growth after hatching is negligible and uses egg numbers and egg development time in the calculations. Therefore, rotifer productivity estimates were restricted to species that carry their eggs. Productivity estimates may be underestimated due to our 2-wk sampling interval and the requirement for rotifer eggs; however, results should be valuable for relative comparisons among ponds. Egg development time is a function of temperature; we used the general regression of Bottrell et al. (1976) to estimate rotifer egg development times.

Zooplankton community nutrient regeneration rates.-Of the $4.0 \mathrm{~L}$ of water sieved to collect rotifers, 3.2 L were filtered through Whatman GF/D filters at $\leq 26.7 \mathrm{kPa}$ negative pressure to remove phytoplankton and detritus-associated bacteria.

A set of 10 vertical net tows was made at each of the three stations per pond and held in a bucket. Live zooplankton were concentrated by slowly pouring both the remaining water-column sample $(\sim 35 \mathrm{~L})$ and the net zooplankton (representing $\sim 90 \mathrm{~L}$ ) onto a $35-\mu \mathrm{m}$ sieve. Zooplankton were gently rinsed from the sieve into the $3.2 \mathrm{~L}$ of filtered water and used for nutrient regeneration and respiration rate analyses. The use of a $35-\mu \mathrm{m}$ sieve was necessary to quantitatively include rotifers (Likens and Gilbert 1970) in nutrient regeneration and respiration rate analyses. The concentration of zooplankton $(\sim 40 \times)$ was needed to obtain detectable nutrient regeneration and respiration rates throughout the study. It should be noted that nutrient regeneration rates obtained after sieving and enclosure at higher densities may not accurately represent in situ conditions. However, procedures were identical among ponds, yielding valid comparisons.

Four clear, acid-washed biological oxygen demand (BOD) bottles were filled with subsamples of mixed zooplankton concentrate, sealed, and suspended in the pond at 0.5 (Secchi depth) for $2-5 \mathrm{~h}$, then collected on a tared Whatman GF/C filter. Filtered water and filters + zooplankton were stored on ice until laboratory analyses. Nutrient regeneration rates were estimated by comparing nutrient levels ( $\mathrm{NH}_{3}-\mathrm{N}$ and SRP) before and after enclosure of zooplankton in situ. Pre- and postenclosure water samples were analyzed for $\mathrm{NH}_{3}-\mathrm{N}$ and $\mathrm{SRP}$ as above. Detection limit for both nutrients was 
$0.02 \mathrm{mg} / \mathrm{L}$. Samples with a calculated nutrient concentration $<0.02 \mathrm{mg} / \mathrm{L}$ were entered in nutrient regeneration calculations as having $0.00 \mathrm{mg} / \mathrm{L}$.

Filters with zooplankton from the in situ enclosures were oven-dried at $60^{\circ} \mathrm{C}$ for $\geq 24 \mathrm{~h}$ and weighed. The quantity of $\mathrm{NH}_{3}-\mathrm{N}$ or $\mathrm{PO}_{4}-\mathrm{P}$ (as SRP) regenerated by the enclosed zooplankton during the enclosure period was expressed as milligrams of nutrient per milligram dry mass per hour.

Zooplankton community respiration rates.-Four respiration rate samples were prepared by filtering 300$\mathrm{mL}$ subsamples of concentrated zooplankton (see $\mathrm{Nu}$ trient regeneration section above) onto Whatman GF/ D filters. Filters were sealed in vials and frozen on dry ice until laboratory analysis (Ahmed et al. 1976).

Respiration rates (in milligrams $\mathrm{O}_{2}$ per milligram dry mass per hour) were analyzed according to the method of Owens and King (1975), which assays activity of the respiratory electron transport system (ETS) in a cell-free extract. Briefly, frozen zooplankton were ground in Teflon-glass grinding tubes and centrifuged to make a cell-free extract. The extract was incubated with NADH and NADPH in the presence of a tetrazolium dye that serves as a terminal electron acceptor and turns red upon reduction. Absorbance at $490 \mathrm{~nm}$ was measured with a Dynatech (Helsinki, Finland) MR600 Microplate Reader. Two blanks were prepared for each pond's set of samples: one for turbidity, the other for background color development. Average zooplankton dry masses from nutrient regeneration samples were used in respiration rate calculations because nutrient regeneration and respiration rate samples were subsampled identically from the concentrated zooplankton in the field. In addition, we calculated volumespecific respiration rates (milligrams $\mathrm{O}_{2}$ per liter per hour) as the product of biomass-specific rates and community biomass (milligrams per liter) estimates.

Data processing.-Respiration rate data were not available for 13 May, 16 December, and 10 February 1989; nutrient regeneration data were not available for the latter two dates. In addition, 6 of 648 zooplankton samples were accidentally lost during the study. Preceding and subsequent sample data were averaged to represent missing data points in statistical analyses. If available (March-October), zooplankton samples from intervening weekly sampling trips were used in averaging. If a species was not present on both preceding and subsequent sampling events, it was considered absent in the missing sample.

\section{Statistical analyses}

We addressed our main question (Do similar communities develop in similar ponds?) by first testing the assumption of environmental similarity among ponds, and then comparing zooplankton communities for structure and function. We also compared patterns of community structure to community function at different scales. We used the same multivariate and uni- variate techniques for environmental, structure, and function data sets to facilitate comparisons of analyses.

Statistical analyses of function variables ended with the 27 January 1989 sampling event. Data (except pH) were $\log$-transformed $\left(\log _{10}[x+1]\right)$ prior to analyses, as suggested by Digby and Kempton (1987) to meet assumptions of parametric statistics. All statistical analyses were conducted using SAS (SAS Institute 1985), and included the following.

1) Multivariate ANOVA (MANOVA) tested for overall differences among ponds for multiple variables simultaneously (e.g., environmental data). Ponds and time were designated as treatments, so a "pond effect" could be detected separately from temporal changes, which were expected (and observed), but of secondary interest. Significant difference by MANOVA is a prerequisite for cluster analysis.

2) Cluster analysis (CA) was used to identify groups of similar ponds for the environment, structure, and function annual data sets (analyzed separately). We also performed CA on the data sets by season, to check for patterns occurring over shorter intervals. We used centroid-linkage clustering on Mahalanobis distances for quantitative data. Mahalanobis distances are useful for data of different scales (Digby and Kempton 1987), and were derived from canonical discriminant analysis (CDA). Clustering was performed only if canonical variates in CDA were significant. Digby and Kempton (1987) considered centroid-linkage clustering to be more resistant to outliers and less likely to produce indistinct clusters than other methods. We selected this method primarily as a stringent test of the environmental similarity assumption: indistinct clusters (i.e., clusters in which points are not closely spaced) would indicate similar environmental conditions among ponds. For consistency, structure (taxa density and biomass) and function (productivity, respiration, and nutrient regeneration) data sets were also clustered by centroid-linkage clustering. An exception was presence/absence data, which we analyzed by single-linkage clustering on percentage similarity values. Singlelinkage (or "nearest neighbor") clustering is more likely to produce indistinct clusters than other methods (Digby and Kempton 1987). Therefore, distinct clusters identified by this method added stringency to our conclusions regarding species richness, which has been the traditional focus of colonization studies.

3) Principal components analysis (PCA; correlation matrix) was used to make temporal trajectories of each pond's multivariate data sets (environment, structure, function data sets for the year, analyzed separately). A plot of trajectories for each pond reduced dimensionality of the multivariate data set while representing much of the variation among ponds through time (Austin 1977).

4) Analysis of variance (ANOVA), with ponds and time as treatments, no replication. As in MANOVA, overall differences among ponds were tested for, sep- 
TABLE 1. Duncan's multiple range test results for environmental variables.

\begin{tabular}{|c|c|c|c|c|c|c|c|c|c|c|c|c|}
\hline \multirow[b]{2}{*}{ Variable } & \multicolumn{12}{|c|}{ Pond ranking } \\
\hline & 1 & 2 & 3 & 4 & 5 & 6 & 7 & 8 & 9 & 10 & 11 & 12 \\
\hline $\mathrm{NH}_{3}-\mathrm{N}$ & $7^{\mathrm{a}}$ & $12^{\mathrm{a}}$ & $10^{\mathrm{a}}$ & $11^{\mathrm{a}, \mathrm{b}}$ & $8^{a, b}$ & $9^{a, b}$ & $6^{\mathrm{b}}$ & $1^{\mathrm{b}}$ & $3^{\mathrm{b}}$ & $5^{b}$ & $4^{\mathrm{b}}$ & $2^{b}$ \\
\hline Secchi depth & $5^{a}$ & $8^{a}$ & $3^{a}$ & $1^{\mathrm{a}, \mathrm{b}}$ & $4^{\mathrm{a}, \mathrm{b}}$ & $6^{\mathrm{b}, \mathrm{c}}$ & $2^{b, c, d}$ & $11^{\mathrm{c}, \mathrm{d}}$ & $10^{\mathrm{c}, \mathrm{d}}$ & $7^{\mathrm{c}, \mathrm{d}}$ & $12^{\mathrm{c}, \mathrm{d}}$ & $9^{c, d}$ \\
\hline Chlorophyll $a$ & $9^{a}$ & $7^{\mathrm{b}}$ & $6^{\mathrm{b}, \mathrm{c}}$ & $12^{\mathrm{b}, \mathrm{c}, \mathrm{d}}$ & $2^{b, c, d}$ & $11^{\mathrm{b}, \mathrm{c}, \mathrm{d}}$ & $4^{b, c, d}$ & $1^{\mathrm{b}, \mathrm{c}, \mathrm{d}}$ & $10^{\mathrm{b}, \mathrm{c}, \mathrm{d}}$ & $3^{\mathrm{c}, \mathrm{d}}$ & $8^{\mathrm{c}, \mathrm{d}}$ & $5^{\mathrm{d}}$ \\
\hline
\end{tabular}

Notes: Ponds are ranked in descending order (left to right) of variable means. Ponds with the same superscript letter were not significantly different $(P=0.05)$. Similar analyses were conducted for alkalinity and conductivity but are omitted here because coefficients of variation were $\leq 4 \%$ and $\leq 3 \%$, respectively.

arately from the expected (and observed) temporal changes that were of secondary interest. Environmental variables with significant differences among ponds were then analyzed with Duncan's multiple range test $(\alpha=0.05)$. Duncan's test was used because its higher probability of Type I error (more likely to detect differences) provided a stringent test of the assumption of environmental similarity. Zooplankton community structure and function variables with significant overall differences were further analyzed with Tukey's HSD test (Sokal and Rohlf 1981).

In addition, we used two other techniques appropriate to specific questions on a data set or relationships among data sets.

5) Dummy-variable regression analysis (DVRA; Kleinbaum et al. 1988) was used to compare species accrual curves among ponds. This analysis allowed us to statistically analyze (cumulative) colonization; colonization curves could not be statistically analyzed otherwise. DVRA is typically used to compare regressions of experimental treatments to controls; we arbitrarily designated Pond 1 as the reference for DVRA.

6) Multiple regressions and standard partial correlation coefficients (SPCC's; Sokal and Rohlf 1981) indicated the relative roles of zooplankton taxa in community function.

\section{RESULTS}

\section{Environmental variables}

Ponds were environmentally similar over the course of the study year. Statistically detected variation among ponds was due to slight, consistent differences in a few variables (e.g., conductivity and alkalinity). Based on the analyses described below, we considered the assumption of environmental similarity among ponds to be valid.

Univariate analyses.-Ponds were not significantly different for 6 of the 11 measured environmental variables (Appendix). Statistically significant differences were detected for conductivity and alkalinity, despite very similar values among ponds: weekly coefficients of variation ( $\mathrm{CV}$ : standard deviation/mean) were $\leq 2 \%$ and $4 \%$, respectively. Three other variables $\left(\mathrm{NH}_{3}-\mathrm{N}\right.$, Secchi depth, and chlorophyll $a$ ) varied more and were significantly different among ponds $(P \leq 0.001$; Appendix). However, Duncan's test identified only one distinct pond for one of the three variables (Table 1), despite Duncan's test's greater probability of Type I error (more likely to detect differences). Ammonia and SRP concentrations were low throughout the study, and Secchi depths were not related to chlorophyll a concentrations (Appendix).

Multivariate analyses. - All three multivariate analyses (PCA, MANOVA, and CA) indicated ponds were generally similar in environmental variables. Any differences detected among ponds were influenced by slight differences detected for conductivity and alkalinity.

Principal components analysis summarized trends in environmental variables for all ponds through the study year (Fig. 1). The first three principal components (PCs) represented $65 \%$ of the variation for 12 variables in 12 ponds over $1 \mathrm{yr}$ (Table 2). Based on the eigenvector loadings (Table 2), we interpreted PC1 as representing general chemical conditions, PC2 as representing seasonal changes, and PC3 as representing nutrient and productivity conditions. Ponds followed similar trajectories in measured environmental variables (Fig. 1). Most progression in PC1 (general chemistry) occurred in the first half of the study period, reflecting the stabilization of several variables after September. The displacement of trajectories in the direction of PC2 represents the common seasonal changes in temperature and dissolved oxygen. Little net change in PC3 occurred at the end of $1 \mathrm{yr}$, but most ponds exhibited a peak in PC3 scores from mid-May through early June, related to changes in several variables at this time: $\mathrm{NH}_{3}$, $\mathrm{NO}_{2}$, and chlorophyll $a$ increased while Secchi depths decreased.

Ponds were significantly different overall for environmental variables by MANOVA $(P<0.0001)$. However, the MANOVA analysis was strongly influenced by conductivity, alkalinity, and ammonia. As in the univariate ANOVAs, MANOVA detected the slight differences among ponds in those few variables (Appendix).

Cluster analysis of environmental data identified two indistinctly clustered groups of ponds (Fig. 2). This result was consistent with univariate analyses: Duncan's tests placed Pond 9 in distinct groups twice, and grouped Ponds 7, 11, and 12 together in every analysis. Again, this statistical grouping was partially based on minor differences among ponds in conductivity and alkalinity (Appendix). Re-analysis without conductiv- 

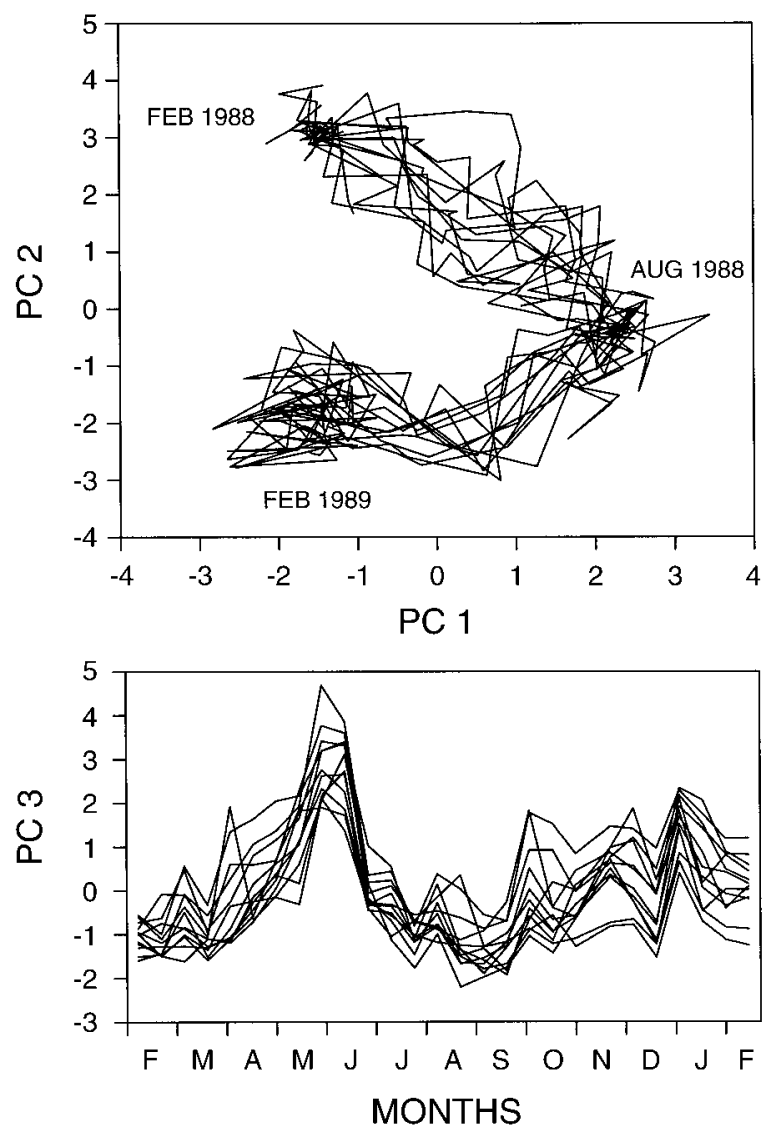

FIG. 1. Principal components trajectories of environmental variables through time. Each line represents a pond. PC 1 represents general chemical conditions, PC 2 represents seasons, PC 3 represents nutrient and productivity conditions. See Table 2 for eigenvectors and eigenvalues.

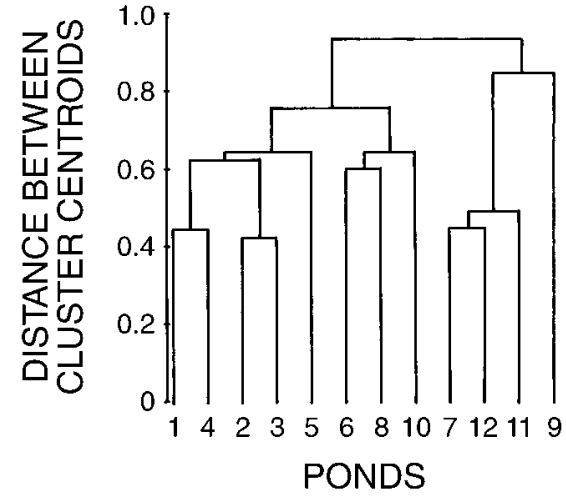

FIG. 2. Cluster analysis of ponds for annual means of environmental variables.

ity and alkalinity data (not shown) provided even less distinct clustering, with only Pond 9 identified as being somewhat different (related to chlorophyll $a$ data). Also, no one pond or set of ponds was consistently unique in cluster analyses per season (Jenkins 1990).

\section{Zooplankton community structure}

Ponds differed in the details of zooplankton community structure (species composition, colonization and accrual curves, and presence/absence patterns). Ponds also varied in aggregated terms (taxa density and biomass). Community-level measures (species richness, density, biomass) indicated less distinct differences among ponds than observed at finer scales.

Species composition.-A total of 61 species were identified during the study, but the cumulative number of species observed per pond ranged from 31 to 39 (Table 3). Any given pond was colonized by one-half to one-third of the apparent regional species pool (i.e., those listed in Table 3). Almost one-half of all species (48\%) were never recorded in $>6$ ponds: of the $52 \%$ recorded in $>6$ ponds, 14 species $(23 \%)$ were recorded in all 12 ponds. Those 14 species may be expected to

TABLE 2. Statistics for principal components analysis of environmental variables. Variables are sorted in order of decreasing absolute value for eigenvectors of PC 1 .

\begin{tabular}{lrrr}
\hline \hline \multirow{2}{*}{$\begin{array}{c}\text { Environmental } \\
\text { variables }\end{array}$} & \multicolumn{3}{c}{ Eigenvectors } \\
\cline { 2 - 4 } & \multicolumn{1}{c}{ PC1 } & \multicolumn{1}{c}{ PC2 } & \multicolumn{1}{c}{ PC3 } \\
\hline $\mathrm{pH}$ & -0.4328 & -0.0530 & 0.0880 \\
$\mathrm{NO}_{3}$-N & 0.4278 & -0.1852 & -0.0945 \\
$\mathrm{Alkalinity}_{\text {Conductivity }}$ & -0.4133 & 0.2196 & 0.0377 \\
Hardness & 0.4014 & 0.1333 & -0.1224 \\
Secchi depth & 0.3365 & 0.3182 & 0.0013 \\
Chlorophyll $a$ & -0.2880 & 0.1168 & -0.4323 \\
$\mathrm{NH}_{3}$-N & -0.2364 & 0.0634 & 0.4850 \\
$\mathrm{NO}_{2}$-N & 0.1680 & 0.0065 & 0.4663 \\
$\mathrm{Soluble}$ reactive phosphorus & 0.0962 & 0.0903 & 0.5692 \\
Dissolved oxygen (1 m) & 0.0812 & -0.1073 & 0.0227 \\
Temperature (1 m) & -0.0339 & -0.6114 & 0.0521 \\
Eigenvalues & 0.0215 & 0.6201 & -0.0091 \\
Proportion of variation (\%) & 3.8738 & 2.3396 & 1.6228 \\
Cumulative proportion $(\%)$ & 32.3 & 19.5 & 13.5 \\
\hline
\end{tabular}


TABLE 3. Ponds colonized by zooplankton species.

\begin{tabular}{|c|c|c|c|c|c|c|c|c|c|c|c|c|c|}
\hline \multirow[b]{2}{*}{ Species } & \multicolumn{12}{|c|}{ Ponds } & \multirow{2}{*}{$\begin{array}{r}\text { Ponds/ } \\
\text { species }\end{array}$} \\
\hline & 1 & 2 & 3 & 4 & 5 & 6 & 7 & 8 & 9 & 10 & 11 & 12 & \\
\hline Anuraeopsis fissa & $\mathrm{x}$ & $\mathrm{x}$ & $\mathrm{x}$ & $\mathrm{x}$ & $\mathrm{x}$ & $\mathrm{x}$ & $\mathrm{x}$ & $\mathrm{x}$ & $\mathrm{x}$ & $\mathrm{x}$ & $\mathrm{x}$ & $\mathrm{x}$ & 12 \\
\hline Brachionus urceolaris & $\mathrm{x}$ & $\mathrm{x}$ & $\mathrm{x}$ & $\mathrm{x}$ & $\mathrm{x}$ & $\mathrm{x}$ & $\mathrm{x}$ & $\mathrm{x}$ & $\mathrm{x}$ & $\mathrm{x}$ & $\mathrm{x}$ & $\mathrm{x}$ & 12 \\
\hline Chaoborus americanus & $\mathrm{x}$ & $\mathrm{x}$ & $\mathrm{x}$ & $\mathrm{x}$ & $\mathrm{x}$ & $\mathrm{x}$ & $\mathrm{x}$ & $\mathrm{x}$ & $\mathrm{x}$ & $\mathrm{x}$ & $\mathrm{x}$ & $\mathrm{x}$ & 12 \\
\hline Chaoborus punctipennis & $\mathrm{x}$ & $\mathrm{x}$ & $\mathrm{x}$ & $\mathrm{x}$ & $\mathrm{x}$ & $\mathrm{x}$ & $\mathrm{x}$ & $\mathrm{x}$ & $\mathrm{x}$ & $\mathrm{x}$ & $\mathrm{x}$ & $\mathrm{x}$ & 12 \\
\hline Conochiloides dossuarius & $\mathrm{x}$ & $\mathrm{x}$ & $\mathrm{x}$ & $\mathrm{x}$ & $\mathrm{x}$ & $\mathrm{x}$ & $\mathrm{x}$ & $\mathrm{x}$ & $\mathrm{x}$ & $\mathrm{x}$ & $\mathrm{x}$ & $\mathrm{x}$ & 12 \\
\hline Eucyclops agilis & $\mathrm{x}$ & $\mathrm{x}$ & $\mathrm{x}$ & $\mathrm{x}$ & $\mathrm{x}$ & $\mathrm{x}$ & $\mathrm{x}$ & $\mathrm{x}$ & $\mathrm{x}$ & $\mathrm{x}$ & $\mathrm{x}$ & $\mathrm{x}$ & 12 \\
\hline Hexarthra mira & $\mathrm{x}$ & $\mathrm{x}$ & $\mathrm{x}$ & $\mathrm{x}$ & $\mathrm{x}$ & $\mathrm{x}$ & $\mathrm{x}$ & $\mathrm{x}$ & $\mathrm{x}$ & $\mathrm{x}$ & $\mathrm{x}$ & $\mathrm{x}$ & 12 \\
\hline Keratella cochlearis & $\mathrm{x}$ & $\mathrm{x}$ & $\mathrm{x}$ & $\mathrm{x}$ & $\mathrm{x}$ & $\mathrm{x}$ & $\mathrm{x}$ & $\mathrm{x}$ & $\mathrm{x}$ & $\mathrm{x}$ & $\mathrm{x}$ & $\mathrm{x}$ & 12 \\
\hline Keratella crassa & $\mathrm{x}$ & $\mathrm{x}$ & $\mathrm{x}$ & $\mathrm{x}$ & $\mathrm{x}$ & $\mathrm{x}$ & $\mathrm{x}$ & $\mathrm{x}$ & $\mathrm{x}$ & $\mathrm{x}$ & $\mathrm{x}$ & $\mathrm{x}$ & 12 \\
\hline Keratella gracilenta & $\mathrm{x}$ & $\mathrm{x}$ & $\mathrm{x}$ & $\mathrm{x}$ & $\mathrm{x}$ & $\mathrm{x}$ & $\mathrm{x}$ & $\mathrm{x}$ & $\mathrm{x}$ & $\mathrm{x}$ & $\mathrm{x}$ & $\mathrm{x}$ & 12 \\
\hline Lecane flexilis & $\mathrm{x}$ & $\mathrm{x}$ & $\mathrm{x}$ & $\mathrm{x}$ & $\mathrm{x}$ & $\mathrm{x}$ & $\mathrm{x}$ & $\mathrm{x}$ & $\mathrm{x}$ & $\mathrm{x}$ & $\mathrm{x}$ & $\mathrm{x}$ & 12 \\
\hline Ostracod & $\mathrm{x}$ & $\mathrm{x}$ & $\mathrm{x}$ & $\mathrm{x}$ & $\mathrm{x}$ & $\mathrm{x}$ & $\mathrm{x}$ & $\mathrm{x}$ & $\mathrm{x}$ & $\mathrm{x}$ & $\mathrm{x}$ & $\mathrm{x}$ & 12 \\
\hline Polyarthra vulgaris & $\mathrm{x}$ & $\mathrm{x}$ & $\mathrm{x}$ & $\mathrm{x}$ & $\mathrm{x}$ & $\mathrm{x}$ & $\mathrm{x}$ & $\mathrm{x}$ & $\mathrm{x}$ & $\mathrm{x}$ & $\mathrm{x}$ & $\mathrm{x}$ & 12 \\
\hline Tropocyclops prasinus & $\mathrm{x}$ & $\mathrm{x}$ & $\mathrm{x}$ & $\mathrm{x}$ & $\mathrm{x}$ & $\mathrm{x}$ & $\mathrm{x}$ & $\mathrm{x}$ & $\mathrm{x}$ & $\mathrm{x}$ & $\mathrm{x}$ & $\mathrm{x}$ & 12 \\
\hline Filinia terminalis & & $\mathrm{x}$ & $\mathrm{x}$ & $\mathrm{x}$ & $\mathrm{x}$ & $\mathrm{x}$ & $\mathrm{x}$ & $\mathrm{x}$ & $\mathrm{x}$ & $\mathrm{x}$ & $\mathrm{x}$ & $\mathrm{x}$ & 11 \\
\hline Monostyla quadridentata & $\mathrm{x}$ & & $\mathrm{x}$ & $\mathrm{x}$ & $\mathrm{x}$ & $\mathrm{x}$ & $\mathrm{x}$ & $\mathrm{x}$ & $\mathrm{x}$ & $\mathrm{x}$ & $\mathrm{x}$ & $\mathrm{x}$ & 11 \\
\hline Alona rustica & $\mathrm{x}$ & $\mathrm{x}$ & & $\mathrm{x}$ & $\mathrm{x}$ & $\mathrm{x}$ & $\mathrm{x}$ & $\mathrm{x}$ & $\mathrm{x}$ & & $\mathrm{x}$ & $\mathrm{x}$ & 10 \\
\hline Bdelloid sp. 1 & $\mathrm{x}$ & $\mathrm{x}$ & $\mathrm{x}$ & & $\mathrm{x}$ & & $\mathrm{x}$ & $\mathrm{x}$ & $\mathrm{x}$ & $\mathrm{x}$ & $\mathrm{x}$ & $\mathrm{x}$ & 10 \\
\hline Bosmina longirostris & $\mathrm{x}$ & $\mathrm{x}$ & $\mathrm{x}$ & $\mathrm{x}$ & $\mathrm{x}$ & $\mathrm{x}$ & $\mathrm{x}$ & & $\mathrm{x}$ & & $\mathrm{x}$ & $\mathrm{x}$ & 10 \\
\hline Lecane aeganea & $\mathrm{x}$ & $\mathrm{x}$ & $\mathrm{x}$ & $\mathrm{x}$ & & $\mathrm{x}$ & $\mathrm{x}$ & $\mathrm{x}$ & & $\mathrm{x}$ & $\mathrm{x}$ & $\mathrm{x}$ & 10 \\
\hline Monostyla bulla & $\mathrm{x}$ & & $\mathrm{x}$ & $\mathrm{x}$ & $\mathrm{x}$ & $\mathrm{x}$ & $\mathrm{x}$ & $\mathrm{x}$ & $\mathrm{x}$ & $\mathrm{x}$ & $\mathrm{x}$ & & 10 \\
\hline Monostyla lunaris & $\mathrm{x}$ & $\mathrm{x}$ & $\mathrm{x}$ & $\mathrm{x}$ & $\mathrm{x}$ & $\mathrm{x}$ & $\mathrm{x}$ & $\mathrm{x}$ & & & $\mathrm{x}$ & $\mathrm{x}$ & 10 \\
\hline Kellicottia bostoniensis & $\mathrm{x}$ & $\mathrm{x}$ & $\mathrm{x}$ & $\mathrm{x}$ & & $\mathrm{x}$ & $\mathrm{x}$ & & & $\mathrm{x}$ & $\mathrm{x}$ & $\mathrm{x}$ & 9 \\
\hline Keratella quadrata & & & $\mathrm{x}$ & $\mathrm{x}$ & $\mathrm{x}$ & & $\mathrm{x}$ & $\mathrm{x}$ & $\mathrm{x}$ & $\mathrm{x}$ & $\mathrm{x}$ & $\mathrm{x}$ & 9 \\
\hline Monostyla elachis & $\mathrm{x}$ & $\mathrm{x}$ & & $\mathrm{x}$ & $\mathrm{x}$ & & & $\mathrm{x}$ & $\mathrm{x}$ & $\mathrm{x}$ & $\mathrm{x}$ & $\mathrm{x}$ & 9 \\
\hline Pleuroxus hamulatus & $\mathrm{x}$ & $\mathrm{x}$ & $\mathrm{x}$ & $\mathrm{x}$ & $\mathrm{x}$ & & $\mathrm{x}$ & & & $\mathrm{x}$ & $\mathrm{x}$ & $\mathrm{x}$ & 9 \\
\hline Trichocerca stylata & $\mathrm{x}$ & $\mathrm{x}$ & $\mathrm{x}$ & $\mathrm{x}$ & & & $\mathrm{x}$ & $\mathrm{x}$ & & $\mathrm{x}$ & $\mathrm{x}$ & $\mathrm{x}$ & 9 \\
\hline Monostyla pyriformis & $\mathrm{x}$ & $\mathrm{x}$ & $\mathrm{x}$ & $\mathrm{x}$ & & & & $\mathrm{x}$ & $\mathrm{x}$ & $\mathrm{x}$ & & $\mathrm{x}$ & 8 \\
\hline Simocephalus serrulatus & & $\mathrm{x}$ & & $\mathrm{x}$ & $\mathrm{x}$ & $\mathrm{x}$ & & $\mathrm{x}$ & $\mathrm{x}$ & & $\mathrm{x}$ & $\mathrm{x}$ & 8 \\
\hline Trichocerca similis & $\mathrm{x}$ & & $\mathrm{x}$ & $\mathrm{x}$ & $\mathrm{x}$ & & $\mathrm{x}$ & & $\mathrm{x}$ & $\mathrm{x}$ & $\mathrm{x}$ & & 8 \\
\hline Chydorus sphaericus & & $\mathrm{x}$ & $\mathrm{x}$ & & $\mathrm{x}$ & $\mathrm{x}$ & $\mathrm{x}$ & $\mathrm{x}$ & & & $\mathrm{x}$ & & 7 \\
\hline Macrocyclops albidus & & $\mathrm{x}$ & & $\mathrm{x}$ & $\mathrm{x}$ & & $\mathrm{x}$ & $\mathrm{x}$ & $\mathrm{x}$ & $\mathrm{x}$ & & & 7 \\
\hline Ascomorpha ovalis & $\mathrm{x}$ & $\mathrm{x}$ & $\mathrm{x}$ & & & $\mathrm{x}$ & $\mathrm{x}$ & & & & & $\mathrm{x}$ & 6 \\
\hline Cephalodella physalis & $\mathrm{x}$ & & $\mathrm{x}$ & & $\mathrm{x}$ & & & & $\mathrm{x}$ & $\mathrm{x}$ & & $\mathrm{x}$ & 6 \\
\hline Bdelloid sp. 2 & & & $\mathrm{x}$ & & & $\mathrm{x}$ & & & $\mathrm{x}$ & $\mathrm{x}$ & & $\mathrm{x}$ & 5 \\
\hline Chaoborus flavicans & & & & & & & & $\mathrm{x}$ & $\mathrm{x}$ & $\mathrm{x}$ & $\mathrm{x}$ & & 4 \\
\hline Conochilus hippocrepis & $\mathrm{x}$ & $\mathrm{x}$ & & $\mathrm{x}$ & $\mathrm{x}$ & & & & & & & & 4 \\
\hline Lecane pusilla & & $\mathrm{x}$ & $\mathrm{x}$ & & $\mathrm{x}$ & $\mathrm{x}$ & & & & & & & 4 \\
\hline Lepadella amphitropis & & & $\mathrm{x}$ & & & $\mathrm{x}$ & $\mathrm{x}$ & $\mathrm{x}$ & & & & & 4 \\
\hline Trichocerca inermis & $\mathrm{x}$ & & & $\mathrm{x}$ & & $\mathrm{x}$ & & & & $\mathrm{x}$ & & & 4 \\
\hline Brachionus angularis & $\mathrm{x}$ & & & & $\mathrm{x}$ & & & & & $\mathrm{x}$ & & & 3 \\
\hline Daphnia parvula & $\mathrm{x}$ & & $\mathrm{x}$ & & & & & & & $\mathrm{x}$ & & & 3 \\
\hline Lecane rhacois & & & & & $\mathrm{x}$ & & $\mathrm{x}$ & & & & $\mathrm{x}$ & & 3 \\
\hline Moina micrura & & & & & & & & & $\mathrm{x}$ & $\mathrm{x}$ & $\mathrm{x}$ & & 3 \\
\hline Trichocerca multicrinis & & & & $\mathrm{x}$ & & & & & $\mathrm{x}$ & & & $\mathrm{x}$ & 3 \\
\hline Cephalodella intuta & & & & $\mathrm{x}$ & & & $\mathrm{x}$ & & & & & & 2 \\
\hline Euchlanis dilatata & $\mathrm{x}$ & & & & $\mathrm{x}$ & & & & & & & & 2 \\
\hline Lecane hornemanni & & & & & $\mathrm{x}$ & & & & & & & $\mathrm{x}$ & 2 \\
\hline Lecane sp. & & & & & $\mathrm{x}$ & $\mathrm{x}$ & & & & & & & 2 \\
\hline Lepadella acuminata & & & & & $\mathrm{x}$ & & & & & $\mathrm{x}$ & & & 2 \\
\hline Trichocerca intermedia & & & $\mathrm{x}$ & & & & & & & $\mathrm{x}$ & & & 2 \\
\hline Trichocerca pusilla & & & $\mathrm{x}$ & $\mathrm{x}$ & & & & & & & & & 2 \\
\hline Trichocerca rattus & & & $\mathrm{x}$ & & $\mathrm{x}$ & & & & & & & & 2 \\
\hline Ascomorpha saltans & & & $\mathrm{x}$ & & & & & & & & & & 1 \\
\hline Bdelloid sp. 3 & & & & & & & & & & & & $\mathrm{x}$ & 1 \\
\hline Colurella sp. & & & & & & & $\mathrm{x}$ & & & & & & 1 \\
\hline Keratella taurocephala & & & & & & & & & & $\mathrm{x}$ & & & 1 \\
\hline Keratella testudo & & & & & $\mathrm{x}$ & & & & & & & & 1 \\
\hline Lecane luna & & & & & & $\mathrm{x}$ & & & & & & & 1 \\
\hline Lecane tenuiseta & & & & & & & $\mathrm{x}$ & & & & & & 1 \\
\hline Monommatta sp. & $\mathrm{x}$ & & & & & & & & & & & & 1 \\
\hline Species per pond & 35 & 31 & 38 & 35 & 39 & 31 & 35 & 30 & 31 & 37 & 33 & 34 & \\
\hline
\end{tabular}


comprise a "core" assemblage common to all ponds, but most of the 14 "core" species rarely existed in all 12 ponds simultaneously (Table 4). Zooplankton community composition varied among ponds due to the three-quarters of the species pool that colonized incompletely, and due to the variable timing of those species that colonized completely.

Zooplankton species varied widely in their colonization success and timing (Table 4). For example, 4 species (and cyclopoid copepod nauplii) were collected $1 \mathrm{wk}$ after the ponds were filled, while other species were collected much later in the year (Table 4). Over one-half of the species were first recorded in a single pond, and only 3 species were first recorded in $>6$ ponds. Of the 478 values shown in Table 4, only 148 $(31 \%)$ are $>6$, indicating that it was unlikely for any given species to exist simultaneously in more than onehalf of the ponds. In addition, values $>6$ in Table 4 tend to be concentrated in selected taxa (e.g., copepods, Chaoborus, ostracods, and Keratella species).

Colonization and accrual curves. - All ponds exhibited increases in species richness several weeks after the ponds were filled, but trends varied among ponds and through time (Fig. 3). Some ponds exhibited a lag in colonization curves (e.g., Ponds 2, 4, 7), while others did not (e.g., Ponds 6, 12). Most ponds exhibited declining species numbers after September, but seasonal declines varied markedly among ponds (Fig. 3). Shortterm changes in species number were not synchronous nor had similar amplitude among all ponds, and did not coincide with environmental changes, including rainstorms. The variation among ponds in colonization curves precluded statistical comparison by dummyvariable regression analysis (DVRA) or analysis of covariance, which require a common model for all curves.

Species accrual (cumulative species richness) was analyzed by DVRA: curves were significantly different among ponds $(P<0.001)$ and ponds differed in estimates of both species accrual rates and numbers accrued (Table 5). A greater accrual rate did not necessarily lead to a greater estimate of species accrued: for example, compare Ponds 6 and 10 in Table 5.

Species presencelabsence.-Ponds were significantly different in species presence/absence pattern by MANOVA $(P<0.0001)$. Cluster analysis of presence/ absence data indicated two major sets of ponds, $57 \%$ similar to each other (Fig. 4). Each cluster included ponds that had similar $(\geq 75 \%)$ species lists. Ponds 8 and 11 were most similar $(85 \%)$. No apparent relationship existed between clusters and spatial position of the ponds (i.e., proximate ponds did not cluster together), and cluster patterns of environmental and species richness data did not coincide (compare Figs. 2 and 4).

Taxa density.-Zooplankton species were grouped into taxa (rotifers, copepods, cladocerans, Chaoborus, and ostracods) for analyses of density and biomass. Rotifers achieved densities $10 \times$ greater than copepods, the next most abundant taxon (Fig. 5). However, peak rotifer densities were brief, and some ponds never achieved rotifer densities exceeding 1000 organisms/ L. Population peaks were seasonal, in that most occurred in midsummer to early autumn, with one major exception: Keratella in Pond 10 exhibited very high densities from December 1988 to January 1989. In addition, major population peaks often occurred in only one or a few ponds. Thus, a genus or species may have been present in all ponds but abundant in only one or a few ponds at any time.

Eucyclops agilis and Tropocyclops prasinus dominated copepod densities throughout the year. A third copepod, Macrocyclops albidus, was also observed at low densities in most ponds late in the study (Table 4). E. agilis and T. prasinus are similar in size and could only be distinguished as adult females. However, the June peak in copepod densities (Fig. 5) appeared to be due to hatching of $E$. agilis nauplii, based on the timing of $E$. agilis adult females with egg sacs. Similarly, the October peaks appeared to be due to $T$. prasinus nauplii. Thus, naupliar cohorts of the two dominant copepod species seem to have been temporally separated.

Daphnia parvula and Moina micrura occurred briefly in a few ponds during May and June, but cladocerans were not observed in most ponds until August (Table 4). Cladocerans reached modest densities in a few ponds from October to February, but no pond contained cladocerans at a level similar to Pond 9, where Bosmina longirostris maintained a dense population from September through January (Fig. 5).

Chaoborus punctipennis was the dominant Chaoborus species: C. americanus and C. flavicans appeared in some samples. Chaoborus populations were roughly synchronous in ponds, exhibiting peak densities during May-June (Fig. 5). Peak densities were due to early instars, and densities generally declined through the summer, presumably due to mortality and emergence.

Ostracods were present in low densities: they were omitted in Fig. 5 but were included in statistical analyses. It is likely that sampling underestimated densities of these littoral/benthic organisms.

Ponds were significantly different in taxa density by MANOVA $(P=0.0001)$. Cluster analysis of taxa density indicated Ponds 8 and 9 were different from other ponds, which otherwise clustered in two groups (Fig. $6)$. This result bears little resemblance to the cluster analysis for species presence/absence (Fig. 4), which is probably related in part to the use of taxa (Rotifera, etc.) vs. species. Principal components analysis of taxa density did not summarize patterns beyond those in Fig. 5.

Taxa biomass. - Trends in taxa biomass (Fig. 7) were generally similar to taxa density (see Fig. 5), with a few exceptions. Rotifer biomass in Pond 8 during January 1989 more than doubled peak values in any other pond during the year due to Hexarthra mira population density. This rotifer is large and consequently contributed greatly to zooplankton community biomass. High 
TABLE 4. Colonization timing and the number of ponds in which each species was collected each week of the study.

\begin{tabular}{|c|c|c|c|c|c|c|c|c|c|c|c|c|c|c|c|c|c|c|}
\hline \multirow[b]{2}{*}{ Species } & \multicolumn{18}{|c|}{ Weeks } \\
\hline & 1 & 3 & 5 & 7 & 9 & 11 & 13 & 15 & 17 & 19 & 21 & 23 & 25 & 27 & 29 & 31 & 33 & 35 \\
\hline Filinia terminalis & 10 & 3 & & 2 & 2 & & & 1 & & & 1 & 2 & & 7 & & 2 & 3 & \\
\hline Keratella quadrata & 8 & 1 & 2 & & 2 & & & 3 & 1 & & & & & & & & & \\
\hline Cyclopoid nauplii & 4 & 5 & 7 & 11 & 10 & 10 & 10 & 11 & 12 & 12 & 12 & 12 & 12 & 12 & 12 & 12 & 12 & 12 \\
\hline Eucyclops agilis & 2 & 6 & 7 & 9 & 10 & 11 & 11 & 11 & 10 & & & & 1 & & 4 & & 12 & 4 \\
\hline Brachionus urceolaris & 1 & & & 3 & 7 & 11 & 12 & 12 & 6 & 2 & 1 & & & & & & & \\
\hline Cephalodella physalis & & & 1 & 2 & 1 & & & 2 & & & 1 & & & & & & & \\
\hline Cephalodella intuta & & & 1 & & & & & & & & & & & & & & & \\
\hline Euchlanis dilatata & & & & & 1 & 1 & & & & & & & & & & & & \\
\hline Chaoborus punctipennis & & & & & & 10 & 9 & 12 & 12 & 12 & 12 & 12 & 12 & 12 & 12 & 12 & 12 & 12 \\
\hline Chaoborus flavicans & & & & & & & 3 & 1 & & & & & & & & & & \\
\hline Daphnia parvula & & & & & & & 1 & 2 & 2 & 1 & & & & & & & & \\
\hline Hexarthra mira & & & & & & & & 4 & 10 & 11 & 10 & 10 & 5 & 6 & 4 & 6 & 7 & 5 \\
\hline Polyarthra vulgaris & & & & & & & & 3 & 7 & 7 & 8 & 11 & 11 & 11 & 11 & 12 & 10 & 8 \\
\hline Conochilus hippocrepis & & & & & & & & 3 & & 1 & 1 & & & 1 & & & & \\
\hline Kellicottia bostoniensis & & & & & & & & 1 & 2 & 3 & 2 & 3 & 6 & 3 & & & & \\
\hline Keratella cochlearis & & & & & & & & 1 & 1 & 2 & 1 & 5 & 6 & 10 & 12 & 8 & 11 & 12 \\
\hline Moina micrura & & & & & & & & 1 & 3 & & & & & & & & & \\
\hline Tropocyclops prasinus & & & & & & & & & 3 & 12 & 12 & 12 & 12 & 12 & 12 & 12 & & 12 \\
\hline Chaoborus americanus & & & & & & & & & 2 & 2 & 10 & 8 & 6 & 4 & 1 & & & 1 \\
\hline Anuraeopsis fissa & & & & & & & & & 1 & 3 & 4 & 6 & 10 & 11 & 10 & 7 & 5 & 7 \\
\hline Trichocerca inermis & & & & & & & & & 1 & & & & & 1 & & 1 & 1 & \\
\hline Keratella testudo & & & & & & & & & 1 & & & & & & & & & \\
\hline Lecane flexilis & & & & & & & & & 1 & 4 & 3 & 3 & 6 & 3 & 1 & 1 & 5 & 9 \\
\hline Monostyla bulla & & & & & & & & & & 2 & & 2 & 5 & 6 & 3 & 2 & & 1 \\
\hline Trichocerca intermedia & & & & & & & & & & 1 & & & & 1 & & & & \\
\hline Lecane aeganea & & & & & & & & & & 1 & & 5 & 1 & & & 4 & 3 & 1 \\
\hline Trichocerca stylata & & & & & & & & & & 1 & 2 & 6 & 5 & 4 & & 1 & & \\
\hline Bdelloid sp. 1 & & & & & & & & & & & 1 & 4 & 2 & 5 & 2 & 4 & 1 & 3 \\
\hline Monostyla pyriformis & & & & & & & & & & & & 2 & 5 & 3 & & 1 & 1 & \\
\hline Conochiloides dossuarius & & & & & & & & & & & & 2 & 4 & 6 & 7 & 10 & 4 & 3 \\
\hline Monostyla lunaris & & & & & & & & & & & & 1 & 2 & 1 & 1 & & & 1 \\
\hline Lepadella acuminata & & & & & & & & & & & & 1 & 1 & & & & & \\
\hline Lepadella amphitropis & & & & & & & & & & & & & 2 & & & & & \\
\hline Monostyla quadridentata & & & & & & & & & & & & & & 5 & 5 & 9 & 1 & \\
\hline Ostracod & & & & & & & & & & & & & & 2 & 4 & 8 & 6 & 6 \\
\hline Alona rustica & & & & & & & & & & & & & & 1 & & 1 & 1 & 2 \\
\hline Ascomorpha ovalis & & & & & & & & & & & & & & 1 & & 1 & 1 & 2 \\
\hline Pleuroxus hamulatus & & & & & & & & & & & & & & 1 & 2 & 2 & 3 & 2 \\
\hline Keratella gracilenta & & & & & & & & & & & & & & & 4 & 8 & 9 & 11 \\
\hline Brachionus angularis & & & & & & & & & & & & & & & 2 & 2 & & \\
\hline Trichocerca similis & & & & & & & & & & & & & & & 2 & 5 & 6 & 6 \\
\hline Monostyla elachis & & & & & & & & & & & & & & & 2 & & 4 & 2 \\
\hline Simocephalus serrulatus & & & & & & & & & & & & & & & 1 & 1 & & 1 \\
\hline Ascomorpha saltans & & & & & & & & & & & & & & & 1 & & & \\
\hline Bdelloid sp. 2 & & & & & & & & & & & & & & & 1 & 1 & & 1 \\
\hline Trichocerca pusilla & & & & & & & & & & & & & & & & 2 & & 1 \\
\hline Macrocyclops albidus & & & & & & & & & & & & & & & & 1 & & 2 \\
\hline Bosmina longirostris & & & & & & & & & & & & & & & & 1 & 2 & 4 \\
\hline Keratella crassa & & & & & & & & & & & & & & & & & 5 & 10 \\
\hline Lecane luna & & & & & & & & & & & & & & & & & 1 & \\
\hline Trichocerca rattus & & & & & & & & & & & & & & & & & 1 & 2 \\
\hline Chydorus sphaericus & & & & & & & & & & & & & & & & & & \\
\hline Keratella taurocephala & & & & & & & & & & & & & & & & & & \\
\hline Lecane rhacois & & & & & & & & & & & & & & & & & & \\
\hline Monommatta sp. & & & & & & & & & & & & & & & & & & \\
\hline Lecane hornemanni & & & & & & & & & & & & & & & & & & \\
\hline Trichocerca multicrinis & & & & & & & & & & & & & & & & & & \\
\hline Lecane sp. & & & & & & & & & & & & & & & & & & \\
\hline Lecane tenuiseta & & & & & & & & & & & & & & & & & & \\
\hline Bdelloid sp. 3 & & & & & & & & & & & & & & & & & & \\
\hline Colurella sp. & & & & & & & & & & & & & & & & & & \\
\hline Lecane pusilla & & & & & & & & & & & & & & & & & & \\
\hline
\end{tabular}

Bosmina longirostris densities in September-January were comparable in biomass to that of less numerous (but larger) cladocerans in other ponds.

Ponds were significantly different for taxa biomass
(MANOVA; $P=0.0001$ ), and again, ponds 8 and 9 clustered apart from other ponds (Fig. 8). However, taxa biomass cluster patterns among other ponds were different than for taxa density (Fig. 6). Principal com- 
TABLE 4. Extended.

\begin{tabular}{|c|c|c|c|c|c|c|c|c|}
\hline \multicolumn{9}{|c|}{ Weeks } \\
\hline 37 & 39 & 42 & 44 & 46 & 48 & 50 & 52 & 54 \\
\hline \multicolumn{9}{|c|}{1} \\
\hline 12 & 12 & 12 & 11 & $\begin{array}{l}9 \\
1\end{array}$ & 11 & $\begin{array}{l}5 \\
1\end{array}$ & 8 & $\begin{array}{r}10 \\
4\end{array}$ \\
\hline 11 & 10 & 2 & 1 & 1 & & & & \\
\hline 5 & $\begin{array}{l}1 \\
2\end{array}$ & 3 & 2 & 1 & 1 & $\begin{array}{l}1 \\
1 \\
1\end{array}$ & 1 & 1 \\
\hline 12 & 12 & 11 & 12 & 12 & 12 & 12 & 12 & 12 \\
\hline $\begin{array}{r}12 \\
1\end{array}$ & 12 & 12 & 12 & 12 & 12 & 10 & 11 & 11 \\
\hline 8 & 8 & 4 & 4 & & & & & \\
\hline $\begin{array}{l}7 \\
2\end{array}$ & 2 & $\begin{array}{l}2 \\
2\end{array}$ & 1 & & 4 & 4 & 5 & 4 \\
\hline 3 & & 2 & & & & & & \\
\hline $\begin{array}{l}1 \\
1\end{array}$ & 1 & 1 & 1 & & 1 & 1 & & \\
\hline \multirow[t]{2}{*}{1} & 4 & 1 & 2 & & 2 & 3 & 1 & 1 \\
\hline & 1 & & & & & 2 & & \\
\hline 6 & 9 & 9 & 8 & 5 & 9 & 8 & 11 & 10 \\
\hline 3 & $\begin{array}{l}3 \\
1\end{array}$ & 5 & 5 & 7 & 7 & $\begin{array}{l}9 \\
3\end{array}$ & $\begin{array}{l}8 \\
3\end{array}$ & $\begin{array}{l}4 \\
1\end{array}$ \\
\hline 3 & 2 & 7 & 6 & 2 & 8 & 5 & 4 & 4 \\
\hline 10 & 8 & 10 & 10 & 10 & 9 & 9 & 6 & 5 \\
\hline $\begin{array}{l}2 \\
1\end{array}$ & 3 & 3 & 1 & & & & & \\
\hline \multirow[t]{2}{*}{3} & 3 & 2 & 4 & 5 & 6 & 4 & 4 & 6 \\
\hline & & & & & 1 & & 1 & \\
\hline 3 & 3 & 6 & 4 & 2 & 4 & 2 & 4 & 5 \\
\hline 4 & 4 & 6 & 4 & 6 & 6 & 6 & 8 & 3 \\
\hline 12 & 11 & 10 & 12 & 12 & 8 & 10 & 6 & 8 \\
\hline \multirow{6}{*}{$\begin{array}{l}2 \\
1\end{array}$} & 1 & 3 & 3 & 3 & 5 & 5 & 6 & 7 \\
\hline & 1 & 1 & 1 & & & 2 & & \\
\hline & & & 1 & & 1 & 1 & 1 & \\
\hline & & & & & 1 & & 1 & \\
\hline & & & & & & $\begin{array}{l}1 \\
1\end{array}$ & & \\
\hline & & & & & & & $\begin{array}{l}1 \\
4\end{array}$ & \\
\hline
\end{tabular}

ponents analysis did not summarize taxa biomass patterns beyond those in Fig. 7 .

Community-level structure.-Taxa density and biomass were each summed to community-level density

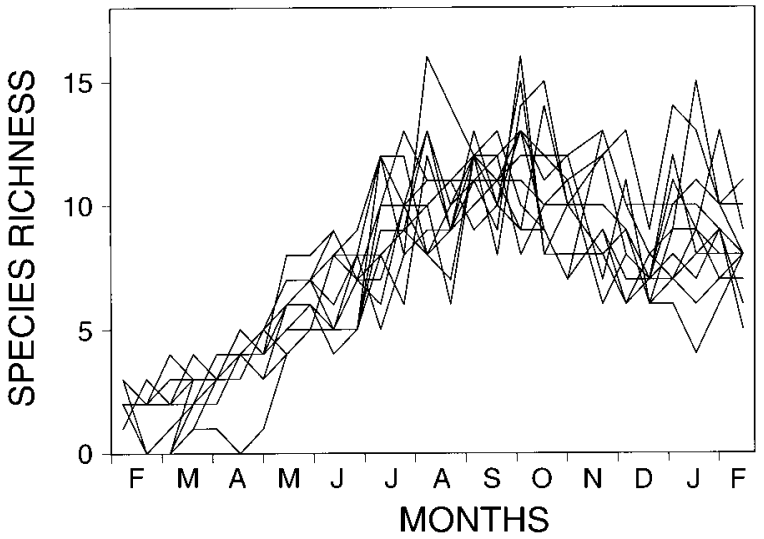

FIG. 3. Zooplankton colonization curves (species richness at each sample date). Each line represents a pond.

and biomass. Ponds were significantly different for community density by ANOVA $(P=0.009)$, although Tukey's HSD identified two widely overlapping groups. Ponds were also significantly different for community biomass (ANOVA; $P=0.04$ ), but Tukey's HSD could not discern separate groups. Ponds were marginally different for species richness (ANOVA; $P=0.07$ ). Multivariate ANOVA of community density, biomass, and species richness detected significant differences among ponds $(P=0.001)$. However, canonical variates in CDA were not significant $(P=0.35)$, precluding meaningful cluster analysis. Finally, PCA of community density, biomass, and species richness yielded patterns similar to those shown in Fig. 3, reflecting the importance of species richness in PCA eigenvectors.

\section{Zooplankton community function}

Zooplankton community function varied through time, but no clear distinctions among ponds could be discerned for community-level productivity, annual community production, community respiration rates, and nutrient regeneration rates. Taxa-level productivity

TABle 5. Species accrual rate ( $r$; species per day) and number of species accrued $(K)$ per pond, derived from dummyvariable regression analysis (Kleinbaum et al. 1988) of logistic regressions (see Statistical analyses for further explanation). All regressions were highly significant $(P<$ 0.001 ) and the lowest $R^{2}$ value $=0.953$. Regression curves were significantly different among ponds $(P<0.001)$.

\begin{tabular}{ccc}
\hline \hline Pond & $r$ and $95 \% \mathrm{CI}$ & $K$ and $95 \% \mathrm{CI}$ \\
\hline 1 & $0.12448 \pm 0.00580$ & $37.47 \pm 1.31$ \\
2 & $0.12796 \pm 0.00667$ & $32.04 \pm 1.82$ \\
3 & $0.11870 \pm 0.00605$ & $38.44 \pm 1.76$ \\
4 & $0.12095 \pm 0.00315$ & $36.85 \pm 0.87$ \\
5 & $0.13012 \pm 0.00586$ & $39.62 \pm 1.90$ \\
6 & $0.13664 \pm 0.00673$ & $30.98 \pm 1.95$ \\
7 & $0.13187 \pm 0.00607$ & $36.50 \pm 1.94$ \\
8 & $0.12101 \pm 0.00335$ & $34.88 \pm 0.86$ \\
9 & $0.11612 \pm 0.00675$ & $33.42 \pm 1.69$ \\
10 & $0.11412 \pm 0.00605$ & $39.81 \pm 1.70$ \\
11 & $0.12521 \pm 0.00634$ & $34.84 \pm 1.80$ \\
12 & $0.13591 \pm 0.00604$ & $36.64 \pm 2.03$ \\
\hline
\end{tabular}




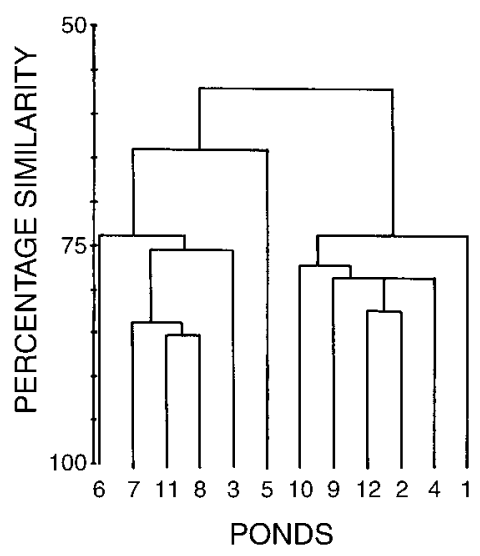

FIG. 4. Cluster analysis of ponds for zooplankton species presence/absence data. Vertical axis represents percent similarity between ponds.

analyses detected differences among ponds, largely based on Cladocera; however, Cladocera made minor contributions to total productivity.

Productivity. - Of the four community function variables measured, only productivity could be analyzed by taxon: all other function measurements were collected for entire communities. Taxa productivity was sporadic (Fig. 9), related to population events (hatching of neonates, emergence, etc.). Ponds were significantly
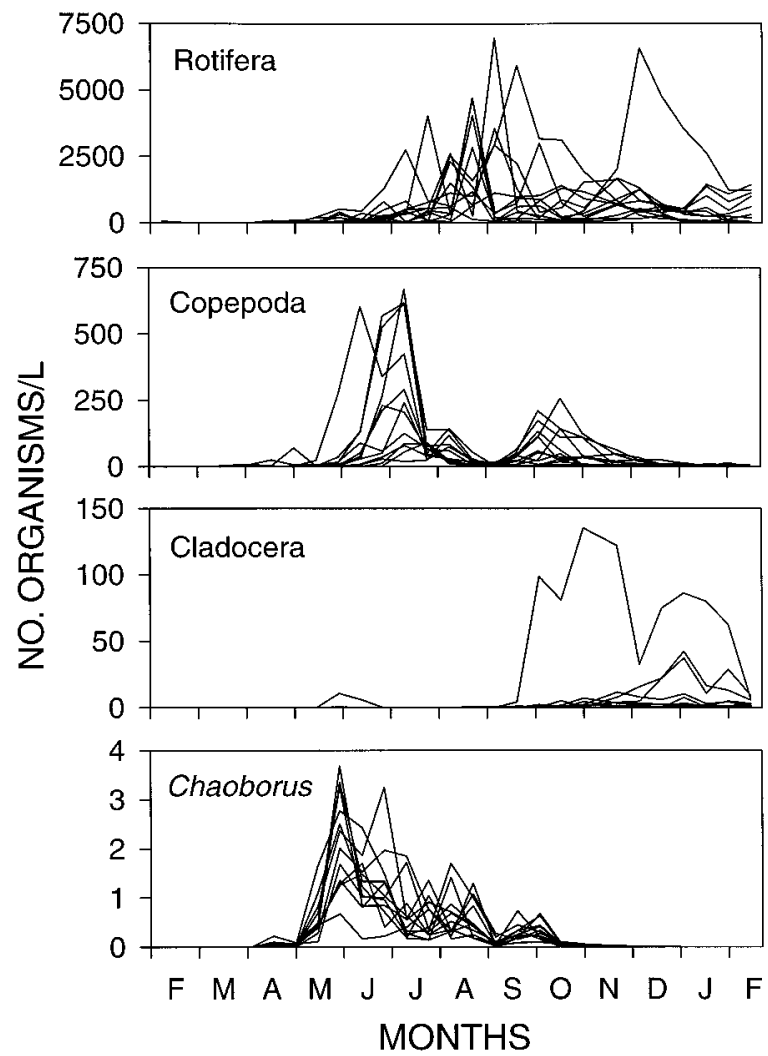

FIG. 5. Zooplankton taxa (Chaoborus, cladoceran, copepod, rotifer) density over time. Each line represents a pond.

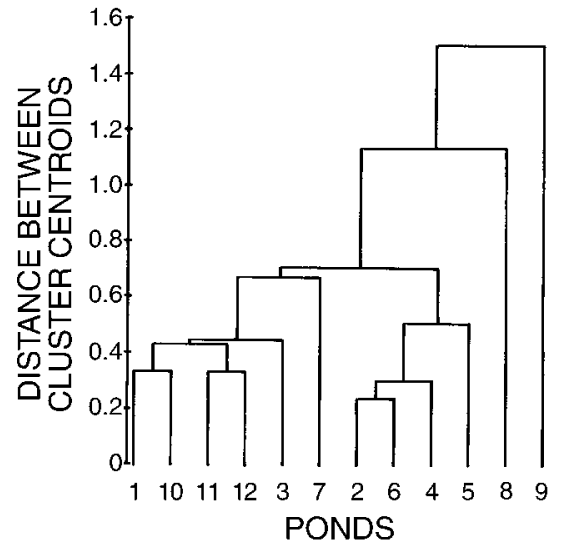

FIG. 6. Cluster analysis of ponds for zooplankton taxa (Chaoborus, cladoceran, copepod, rotifer) density.

different for taxa productivity by MANOVA $(P=$ 0.0001 ), and cluster analysis showed ponds 5 and 8 were different from other ponds, which clustered into two other groups (Fig. 10). This pattern was related to greater cladoceran productivity in pond 5 and 8 than other ponds. PCA of taxa productivity revealed little due to a weak correlation matrix (maximum correlation coefficient $=0.19$ ).

Zooplankton community productivity varied through time (Fig. 11), but was not significantly different

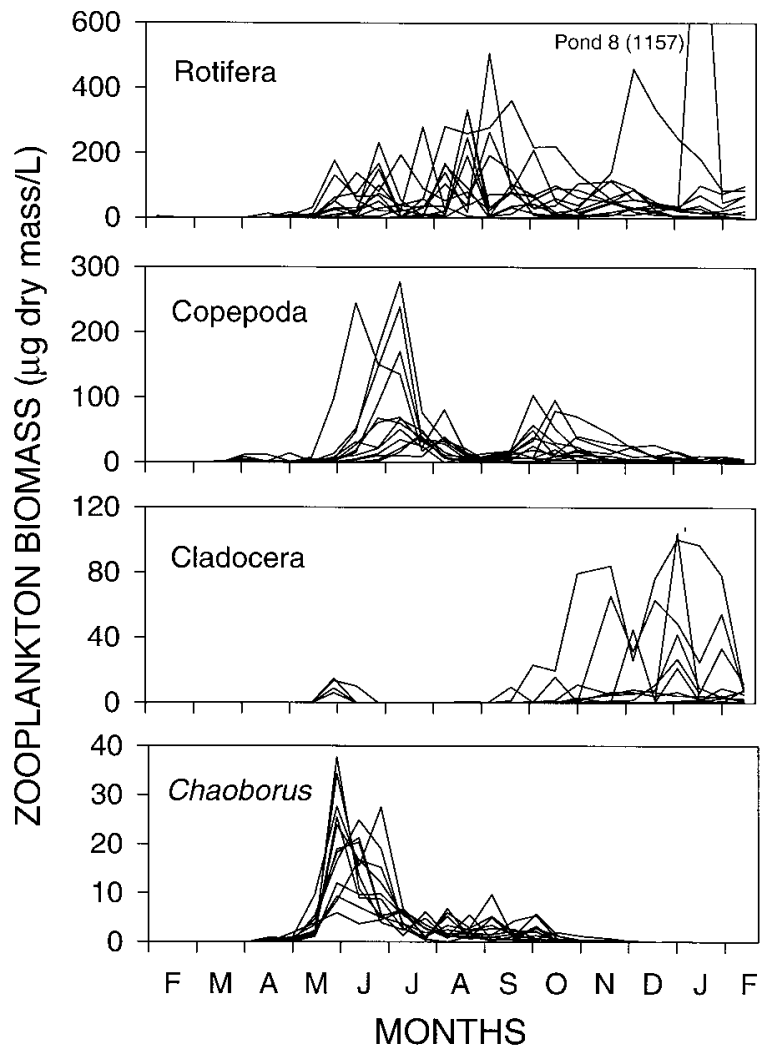

FIG. 7. Zooplankton taxa (Chaoborus, cladoceran, copepod, rotifer) biomass over time. Each line represents a pond. 


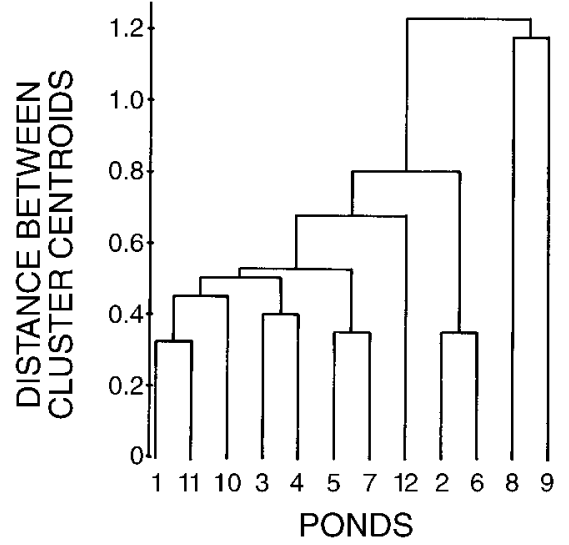

FIG. 8. Cluster analysis of ponds for zooplankton taxa (Chaoborus, cladoceran, copepod, rotifer) biomass.

among ponds (ANOVA; $P=0.52$ ). Community productivity maxima corresponded to peak productivity by various species. Zooplankton community productivity peaked almost synchronously for all ponds on three occasions: late May, late June, and early September. May and June peaks were due to Chaoborus and copepod populations in the ponds. Early September peaks were due to Chaoborus emergence and several rotifer taxa, especially Anuraeopsis, Keratella, and Polyar-
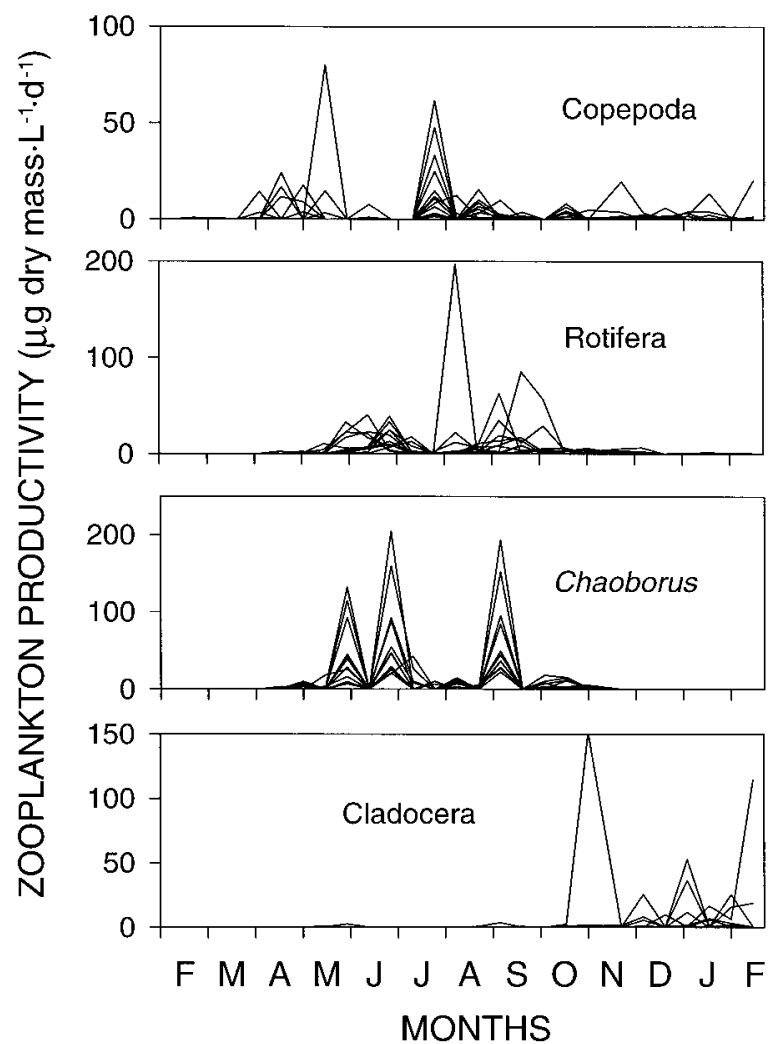

FIG. 9. Zooplankton taxa (Chaoborus, cladoceran, copepod, rotifer) productivity over time. Each line represents a pond.

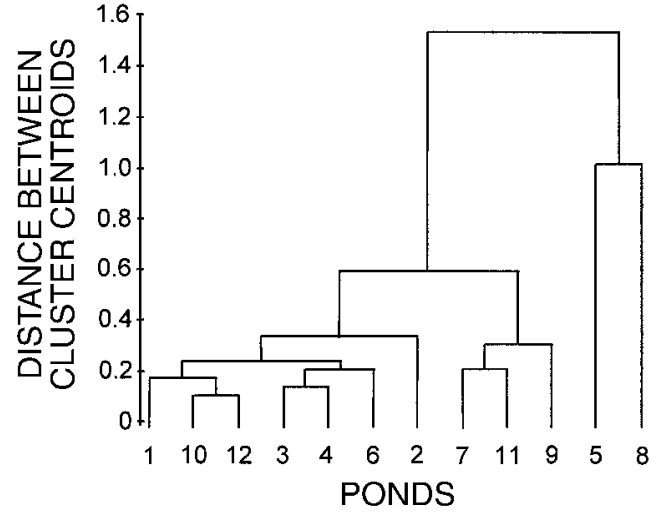

Fig. 10. Cluster analysis of ponds for zooplankton taxa (Chaoborus, cladoceran, copepod, rotifer) productivity.

thra. At other times of the year, zooplankton community productivity was low in most ponds (Fig. 11).

Annual zooplankton community production values ranged from 3.8 to $9.7 \mathrm{mg}$ dry mass/L (Table 6), but ponds were not significantly different in total production $(P=0.52$; ANOVA $)$.

Respiration rates.-Biomass-specific respiration rates roughly followed an annual cycle (Fig. 12). Respiration rates were adjusted for temperature, so this seasonal effect on community respiration rates was not a direct effect of temperature on poikilothermic metabolic rates. Volume-specific rates also followed an annual cycle, but with markedly different patterns among ponds, due to the additional influence of zooplankton biomass in calculations (Fig. 12). Analyses below refer to biomass-specific respiration rates.

Community respiration rates were significantly different among ponds ( $P=0.0001$; ANOVA $)$, but ponds were separated into two widely overlapping groups: ponds $9,4,8,1,10,6$, and ponds $4,8,1,10,6,5,2$, 7, 3, 12, 11 ( $P=0.05$; Tukey's HSD). In addition, variation among ponds was lower for respiration than other functional measures (Jenkins 1990).

Ammonia regeneration rates.-Some ponds exhib-

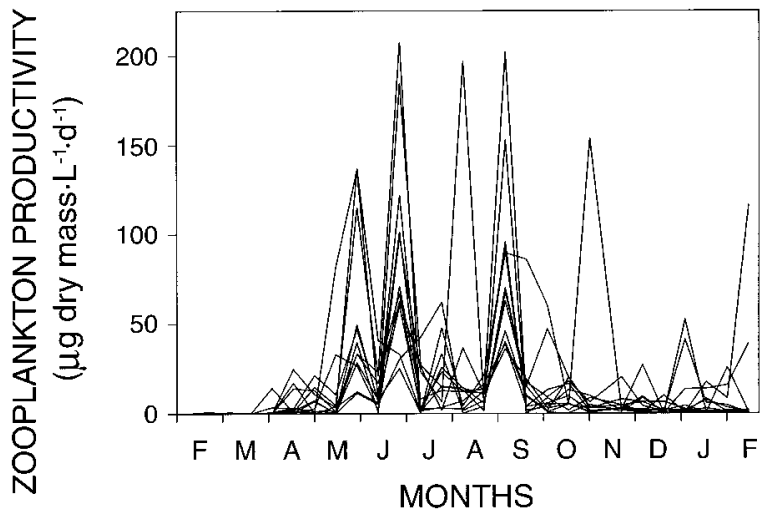

FIG. 11. Zooplankton community productivity over time. Each line represents a pond. 
TABLE 6. Major zooplankton taxa annual production (mg dry mass/L) and percentage of total annual production (in parentheses).

\begin{tabular}{cccccc}
\hline \hline Pond & Chaoborus & Copepoda & Rotifera & Cladocera & Total \\
\hline 1 & $1863(43.1)$ & $680(15.7)$ & $1735(40.1)$ & $44(1.0)$ & 4323 \\
2 & $2116(44.6)$ & $552(11.6)$ & $2076(43.7)$ & $4(0.1)$ & 4748 \\
3 & $4441(67.6)$ & $964(14.7)$ & $1143(17.4)$ & $23(0.4)$ & 6571 \\
4 & $4904(63.0)$ & $1548(19.9)$ & $1305(16.8)$ & $27(0.4)$ & 7784 \\
5 & $3014(33.7)$ & $225(2.5)$ & $4217(47.1)$ & $1488(16.6)$ & 8943 \\
6 & $1713(44.8)$ & $816(21.3)$ & $1274(33.3)$ & $22(0.6)$ & 3825 \\
7 & $3306(66.6)$ & $414(8.3)$ & $1070(21.5)$ & $176(3.5)$ & 4966 \\
8 & $2162(22.3)$ & $1052(10.8)$ & $2209(22.8)$ & $4279(44.1)$ & 9702 \\
9 & $2830(52.1)$ & $1013(18.6)$ & $763(14.0)$ & $828(15.2)$ & 5434 \\
10 & $4980(71.1)$ & $800(11.4)$ & $1170(16.7)$ & $51(0.7)$ & 7000 \\
11 & $1772(40.8)$ & $1103(25.4)$ & $809(18.6)$ & $658(15.2)$ & 4343 \\
12 & $3688(65.2)$ & $735(13.0)$ & $1131(20.0)$ & $100(1.8)$ & 5654 \\
Average & $3066(51.2)$ & $825(14.4)$ & $1575(26.0)$ & $642(8.3)$ & 6108 \\
1 SD & $1159(15.5)$ & $334(6.2)$ & $907(11.8)$ & $1181(13)$ & 1828 \\
\hline
\end{tabular}

ited synchronous peaks in $\mathrm{NH}_{3}-\mathrm{N}$ regeneration rates, but that synchrony appeared to be less uniform among ponds than peaks in productivity and respiration rates. All ponds had low $\mathrm{NH}_{3}-\mathrm{N}$ regeneration rates until June, and then most exhibited increases in $\mathrm{NH}_{3}-\mathrm{N}$ regeneration. A few ponds shared similar timings during summer and most ponds again exhibited peaks in $\mathrm{NH}_{3}-\mathrm{N}$ regeneration rates during November.

Zooplankton community $\mathrm{NH}_{3}-\mathrm{N}$ regeneration rates varied through time (Fig. 13), but ponds were not significantly different $(P=0.17$; ANOVA). Variation among ponds was inconsistent through time.

Phosphate regeneration rates.-Most detectable

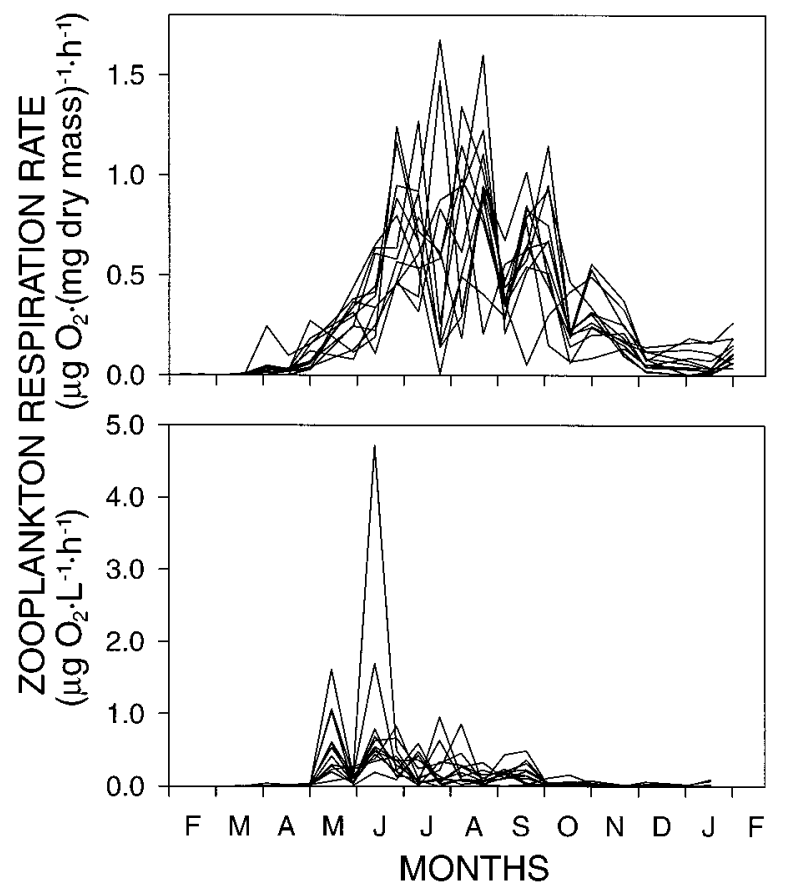

FIG. 12. Zooplankton community respiration rates over time, expressed per unit dry mass (top) and per liter (bottom). Each line represents a pond.
$\mathrm{PO}_{4}-\mathrm{P}$ regeneration rates occurred in the first half of the study year: $\mathrm{PO}_{4}-\mathrm{P}$ levels were low during most of the study and zooplankton usually did not produce sufficient quantities during in situ incubations to reach detectable levels. Ponds were not significantly different in $\mathrm{PO}_{4}-\mathrm{P}$ regeneration rates $(P=0.30$; ANOVA), due to inconsistent and substantial temporal variation among ponds.

Multivariate analyses of community-level function.-Ponds were significantly different by MANOVA $(P=0.004)$, due primarily to variation among ponds in respiration rates. However, canonical variates in CDA were not significant $(P=0.79)$ to serve as a basis for cluster analysis. Principal component analysis (PCA) of community-level function indicated roughly similar, seasonal trends in all ponds over time (Fig. 14).

\section{Structure-function comparisons}

We compared zooplankton community structure and function data (presented separately above) for patterns

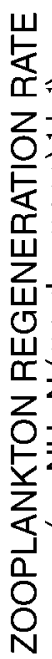

岁

FIG. 13. Zooplankton community $\mathrm{NH}_{3}-\mathrm{N}$ regeneration rates over time. Each line represents a pond. 


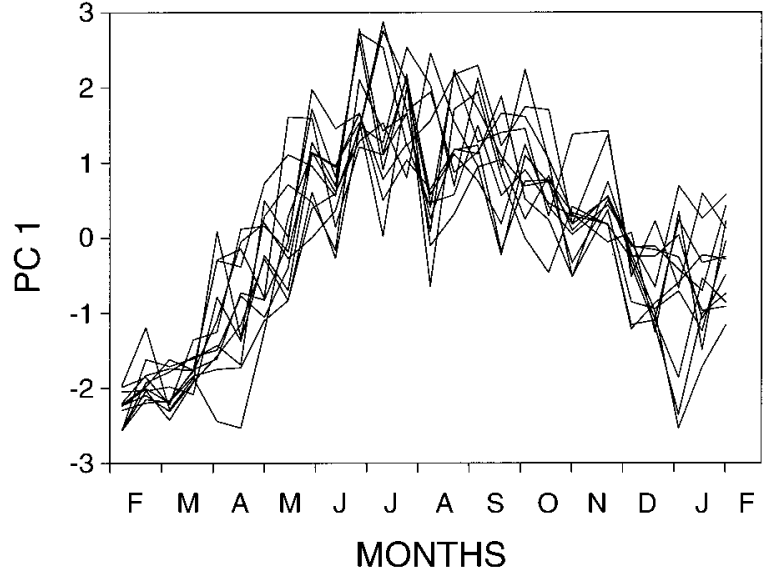

FIG. 14. Principal component 1 trajectories of zooplankton community function. Each line represents a pond. Eigenvalue $=1.77$ (59\% of variation). Eigenvectors: productivity $=0.61$, respiration $=0.67, \mathrm{NH}_{3}-\mathrm{N}$ regeneration $=0.42$. $\mathrm{PO}_{4}-\mathrm{P}$ regeneration was not included in this analysis because most values were below the detection limit (see Zooplankton community function: Phosphate regeneration rates).

among ponds. Details of zooplankton community structure consistently indicated that ponds were different, but function data could not be collected at equivalent levels of detail. Therefore, we compared structure and function at two scales of organization: taxa-level (Chaoborus, copepod, rotifer, cladoceran) and community-level. Community-level structure was evaluated as total biomass, total density, and species richness; community-level function was evaluated as respiration, total productivity, and $\mathrm{NH}_{3}-\mathrm{N}$ regeneration rates. In addition, we examined the relative importance of community structure components in community function by comparing taxa biomass to community function variables.

Analyses of taxa-level structure (density, biomass) and function (productivity) identified distinct sets of ponds, but neither of the structure patterns (Figs. 6 and 8) corresponded to the patterns identified by function (Fig. 10). In addition, taxa structure and function varied among ponds and at comparable levels of variance throughout the study (Fig. 15). However, some structure measures were less variable than corresponding function measures; for example, compare copepod and rotifer density to productivity in Fig. 15.

Analyses of community-level structure and function detected differences among ponds, but could not identify distinct sets of ponds, as taxa-level analyses did. Therefore, greater hierarchical scale reduced the ability to discern clear differences among zooplankton communities for both structural and functional variables. In addition, community-level structure and function were comparable in variation among ponds (Fig. 16).

Zooplankton community composition was related to community function, and the relationships varied among ponds. The relative importance of zooplankton

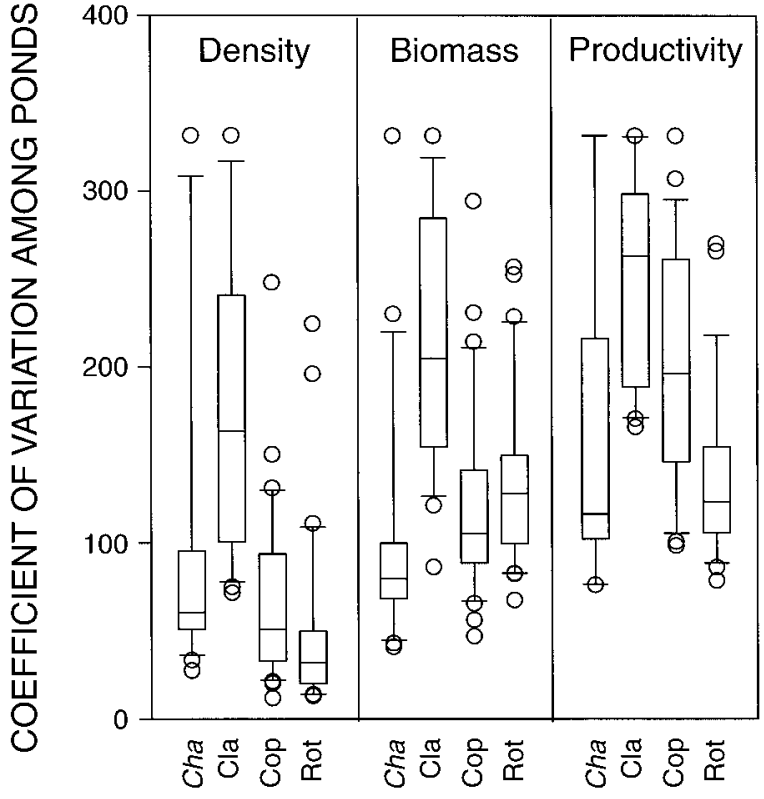

FIG. 15. Distributions of weekly coefficients of variation among ponds for taxa structure (density and biomass) and function (productivity). Cha $=$ Chaoborus, $\mathrm{Cla}=$ cladoceran, Cop $=$ copepod, Rot $=$ rotifer. The horizontal bar in each box indicates the mean, upper and lower limits of the box indicate $\pm 10 \%$, and vertical bars indicate $\pm 90 \%$ of distribution, respectively.

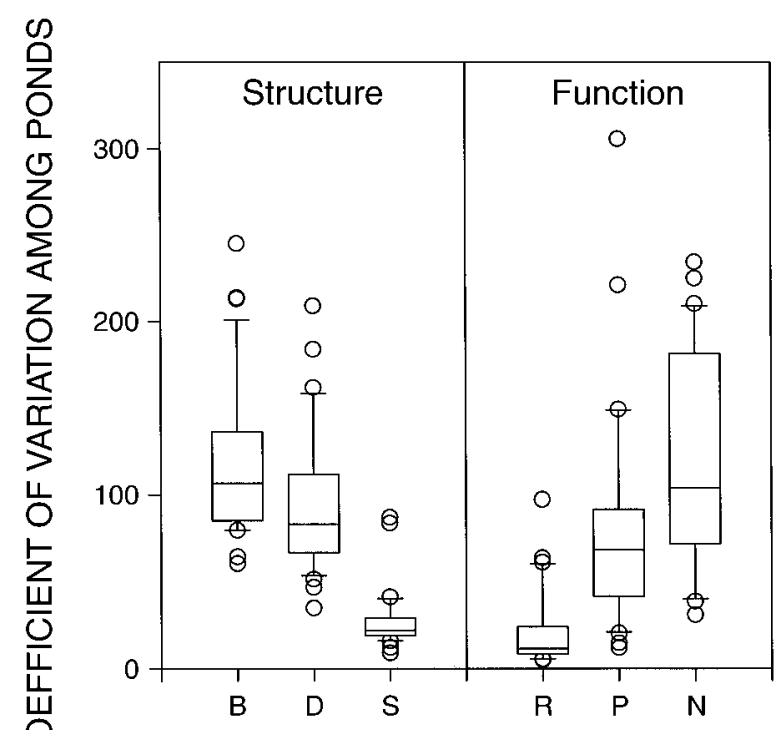

FIG. 16. Distributions of weekly coefficients of variation among ponds for community-level structure and function. Structure measurements: $\mathrm{B}=$ biomass, $\mathrm{D}=$ density, $\mathrm{S}=$ species richness. Function measurements: $\mathrm{R}=$ respiration, $\mathrm{P}$ $=$ productivity, $\mathrm{N}=\mathrm{NH}_{3}-\mathrm{N}$ regeneration. Box plots are as in Fig. 15. 
TABLE 7. Multiple regression statistics for zooplankton taxa biomass relative to community respiration and $\mathrm{NH}_{3}-\mathrm{N}$ regeneration rates. Values are standard partial correlation coefficients, which represent the correlation coefficient expected for a taxon, with all other taxa held constant. Asterisks indicate significant regression $(P<0.05)$.

\begin{tabular}{crrrrrr}
\hline \hline & \multicolumn{5}{c}{ Zooplankton taxa } & \multirow{2}{*}{$\begin{array}{c}\text { Regression } \\
R^{2}\end{array}$} \\
\cline { 2 - 5 } Pond & Copepoda & Chaoborus & Rotifera & Cladocera & Ostracoda & \\
\hline Respiration & & & & & & \\
1 & 0.474 & 0.201 & 0.467 & -0.208 & -0.026 & $0.794^{*}$ \\
2 & 0.366 & 0.195 & 0.518 & 0.067 & 0.030 & $0.75^{*}$ \\
3 & 0.519 & 0.338 & 0.224 & -0.059 & 0.048 & $0.756^{*}$ \\
4 & 0.572 & 0.445 & 0.246 & 0.209 & 0.089 & $0.776^{*}$ \\
5 & 0.125 & 0.378 & 0.703 & -0.036 & 0.033 & $0.82^{*}$ \\
6 & 0.266 & 0.240 & 0.516 & -0.097 & 0.078 & $0.725^{*}$ \\
7 & 0.422 & 0.424 & 0.321 & 0.301 & 0.018 & $0.783^{*}$ \\
8 & 0.484 & 0.349 & 0.373 & 0.065 & 0.118 & $0.784^{*}$ \\
9 & 0.582 & 0.131 & 0.364 & 0.076 & 0.141 & $0.784^{*}$ \\
10 & 0.522 & 0.227 & 0.412 & 0.075 & -0.057 & $0.839^{*}$ \\
11 & 0.271 & 0.501 & 0.434 & -0.126 & 0.105 & $0.812^{*}$ \\
12 & 0.489 & 0.434 & 0.090 & -0.178 & -0.001 & $0.712^{*}$ \\
$\mathrm{NH}_{3}$-N regen & & & & & & \\
1 & -0.151 & -0.183 & 0.477 & -0.052 & -0.216 & 0.14 \\
2 & 0.625 & -0.403 & 0.406 & -0.025 & -0.110 & $0.631^{*}$ \\
3 & 0.533 & -0.119 & 0.239 & -0.094 & -0.116 & $0.453^{*}$ \\
4 & 0.529 & 0.177 & -0.079 & -0.002 & -0.036 & 0.286 \\
5 & 1.029 & -0.278 & -0.247 & -0.146 & -0.029 & $0.673^{*}$ \\
6 & 0.358 & -0.023 & 0.222 & -0.001 & 0.094 & 0.325 \\
7 & 0.356 & 0.063 & -0.187 & -0.348 & -0.264 & 0.285 \\
8 & -0.101 & 0.517 & 0.012 & 0.931 & -0.715 & 0.324 \\
9 & 0.714 & -0.196 & -0.084 & -0.123 & -0.140 & $0.460^{*}$ \\
10 & 0.446 & -0.074 & -0.146 & -0.172 & 0.474 & 0.389 \\
11 & 0.580 & -0.207 & 0.282 & -0.566 & -0.136 & $0.442^{*}$ \\
12 & 0.498 & 0.017 & -0.098 & -0.247 & 0.016 & 0.278 \\
\hline
\end{tabular}

taxa in community production varied among ponds (Table 6). Chaoborus was usually the greatest producer despite its relatively brief period of peak density. Chaoborus larvae attained far greater size than most other zooplankton species, thus producing more biomass at low density than other, more numerous species.

Despite their small size, rotifers were also important producers (Table 6). Annual rotifer production was comparable to or exceeded Chaoborus production in several ponds (e.g., Ponds 1, 2, 5, and 8). Rotifer production exceeded cladoceran production in all but two ponds (Ponds 8 and 9) and was greater than copepod production in all but three ponds (Ponds 4, 9, and 11). Copepod production exceeded cladoceran production in all but two ponds (Ponds 5 and 8; Table 6). Greater cladoceran production in these ponds, especially Pond 8 , was due to persistent populations of the relatively large cladoceran, Simocephalus serrulatus.

Zooplankton community composition was also related to community respiration rates: copepod, Chaoborus, and rotifer biomass correlated with community respiration rates for all ponds (Table 7). Multiple regressions of taxa biomass and community $\mathrm{NH}_{3}-\mathrm{N}$ regeneration rates were significant for only 5 of the 12 ponds (Table 7), and copepods were the most important taxa in those five regressions. Biomass : $\mathrm{NH}_{3}-$ regeneration regressions were not exceptionally strong (mean $R^{2}=0.39$ ), and zooplankton taxa biomass ac- counted for less variation in $\mathrm{NH}_{3}-\mathrm{N}$ regeneration rates than it did for respiration rates. Not surprisingly, multiple regressions of zooplankton biomass against $\mathrm{PO}_{4}^{-}$ $P$ regeneration rates were not significant for 10 of the 12 ponds, and so are omitted from Table 7.

\section{DISCUSSION}

Did similar zooplankton communities develop in similar ponds? Before addressing the question, one must accept the assumption that ponds were environmentally similar. Overall, univariate and multivariate analyses indicated common trends through the year, with minor variation among ponds for some variables, sometimes. Principal components analysis showed all ponds followed the same general trajectory, with minor, transient variation among ponds. Cluster analysis failed to identify distinct clusters, and the weak clusters depended on variables with minor differences among ponds (conductivity, alkalinity). Therefore, we considered the assumption of environmental similarity to be valid for overall comparisons of zooplankton community structure and function.

The simple question (above) cannot be simply answered: our answer depends on the type of data considered. The ponds differed in the details of zooplankton community structure, as measured by both diversity-based (colonization and accrual curves, presence/ absence, species richness) and population-based data 
(density and biomass). However, larger scale measures (taxa, entire community) were not different among ponds. Zooplankton community function was also measured at taxa- and community-levels, and was comparable to equivalent-scale community structure measures in detecting differences among pond communities.

We think our results reveal an overlooked process regulating zooplankton community structure, help illustrate the relationship between zooplankton community structure and function, and are relevant to understanding ecological community assembly in general.

\section{Zooplankton community structure}

Species richness and colonization.-The environmentally similar ponds had different colonization histories. Some species were common to all or most ponds, but many occurred in few ponds, and with mixed timing. Consequently, zooplankton community composition varied among ponds, and colonization and accrual curves differed. These results also indicate that our repeated sampling did not readily transfer organisms among ponds.

Much of aquatic ecology has focused on two sets of processes regulating community structure: interspecific interactions (e.g., Kerfoot and Sih 1987, MacIsaac and Gilbert 1991, Carpenter 1988), and resource constraints (e.g., Lampert 1985). This focus on local-scale processes assumes that community composition is not already limited by regional-scale processes of dispersal (Roughgarden et al. 1987). Few studies have been conducted on zooplankton dispersal and colonization, and those have been limited in scope and/or duration (Maguire 1963, Proctor 1964, Proctor and Malone 1965, Proctor et al. 1967). Nonetheless, zooplankton are typically thought to disperse readily, based on their wide distributions, parthenogenetic life cycles and diapaused stages (King 1980, Brown and Gibson 1983, Wetzel 1983, Pennak 1989, Begon et al. 1990, Lampert and Sommer 1997).

All zooplankton do not disperse readily. Zooplankton species vary widely in colonization rates, and this variance affected species richness and community composition in the young ecosystems. Recent genetic analyses indicate that zooplankton dispersal remains limited among older, isolated water bodies as well (e.g., Boileau and Hebert 1991, Berg and Garton 1994). Also, consider zooplankton that were absent in the experimental ponds during the study (e.g., Synchaeta, Ceriodaphnia, Diaptomus); what might be the effects of slow-colonizing, native species on future community structure? Recent invasions by exotic zooplankton are reminders of the potential importance that rare dispersal may bear on community structure, even in systems as large and old as the Great Lakes (e.g., Sprules et al. 1990).

Therefore, dispersal would appear to be a rate-limiting process of zooplankton community composition, and constitutes an overlooked process regulating zooplankton communities. How important might dispersal be, relative to the more commonly discussed local processes of physical-chemical tolerance and biotic interactions? Paradoxically, dispersal will be most important when it occurs at low rates (Robinson and Edgemon 1988; see Community assembly below). Dispersal will be most important as a factor regulating species diversity, and therefore community structure. Dispersal is unlikely to be more important than planktonic reproduction and the sediment "egg bank" (De Stasio 1991) as a source of organisms for an established population. Dispersal would probably have greatest impact on community composition when it involved influential species (e.g., predators, dominant competitors), but little more can be said without experimental studies of zooplankton introductions.

Density and biomass.-Zooplankton communities consist of populations with a wide range of life histories (Hutchinson 1967), which contributed to an expected seasonal succession of population maxima and temporal variation in zooplankton communities. More importantly, we wished to compare ponds' seasonal successions. As discussed above, zooplankton have been assumed to disperse readily. Also, species-area hypotheses (Williams 1943, MacArthur and Wilson 1967, Connell 1978, Coleman 1981) would predict that samesized, closely spaced, and environmentally similar ponds should receive propagules similarly. Therefore, timing of zooplankton populations in ponds should be similar. In addition, environmentally similar ponds should permit zooplankton populations to develop similar maximum density or biomass.

Copepod and Chaoborus populations were roughly synchronous among ponds, consistent with the rapid colonization observed by copepods and expected for winged insects. Also, copepods and Chaoborus populations develop as cohorts in response to photoperiod and temperature (Parma 1969, Hairston et al. 1990). However, copepod and Chaoborus population maxima were not similar in all ponds. In addition, rotifer and cladoceran populations were far from synchronous and varied in population maxima. Unlike copepods and Chaoborus, rotifers and cladocerans reproduce continuously, making recognition of cohorts difficult. However, asynchrony among rotifer and cladoceran populations was not a simple function of phase-shifted, but similar, population maxima in all ponds. Many species exhibited marked peaks in one or a few ponds without exhibiting peaks in other ponds.

Species that were ubiquitous but dense in only a few ponds were apparently responding to unknown, favorable environmental conditions in those few ponds. Phytoplankton communities also varied among ponds in terms of taxa present, density, and seasonal successions (Rosenzweig and Buikema 1994). However, phytoplankton data were analyzed to genera and each month; phytoplankton community assembly cannot be assessed 
at sufficient resolution to detect its effects on zooplankton. Some zooplankton may have responded to growth of certain phytoplankton in some ponds, perhaps representing a residual, indirect effect of transient environmental differences among ponds on zooplankton densities. Fish were not present in ponds, but invertebrate predators (e.g., Chaoborus) surely affected rotifer and crustacean populations. However, predation by insects did not clearly generate zooplankton density differences among ponds because insects were well dispersed (Jenkins 1990, Layton and Voshell 1993).

Relative importance of zooplankton taxa.-Comparisons of zooplankton taxa for species number, density, and biomass clearly showed that rotifers dominated zooplankton community structure in the first year. Others have found similar results in much older sites, ranging from an oligotrophic lake (Makarewicz and Likens 1979) to a eutrophic reservoir (Pace and Orcutt 1981).

Copepods were present in some ponds at the first sampling date ( $1 \mathrm{wk}$ after filling) and persisted throughout the study, but only briefly rivalled rotifers for dominance. Cladocera never attained an important role in density or biomass. Chaoborus did not contribute substantially to zooplankton density, biomass, or species richness. Ostracods were enumerated collectively and biomass estimates were only approximate, but were not recorded in samples until autumn and were never common.

\section{Zooplankton community function}

Zooplankton community function was dependent on community structure: population events (e.g., hatching, cohort growth, etc.) and community composition affected the timing and magnitude of function data values. Ponds varied considerably in taxa- and community-level function at any given time, but temporal patterns in function were more pronounced than the inconsistent patterns among ponds. Therefore, clear differences among ponds could not be detected for taxa and community function.

A number of studies have evaluated zooplankton in experimental ponds (e.g., Hall et al. 1970, deNoyelles et al. 1982, Giddings et al. 1984, Crossland and Wolff 1985), but most studies have focused on pesticide effects on community structure. To our knowledge, only Hall et al. (1970) evaluated zooplankton community function (production) in multiple experimental ponds: they indicated experimental ponds left unmanipulated for $2 \mathrm{yr}$ were heterogeneous, but presented no data for those 2 yr. Our results suggest that colonization dynamics contributed to that heterogeneity. Our annual zooplankton production estimates are similar to those of Hall et al. (1970) in both range and variability. Little else can be compared between their data and ours for the purpose of examining variation among ponds.

Zooplankton community respiration rates in the ponds were generally lower than values obtained in Lampert's (1984) laboratory studies. We used a dif- ferent method than Lampert, which may contribute to different observed rates. Also, a variety of exogenous factors can affect respiration rates, including light, food, and physico-chemistry (Lampert 1984). Because many zooplankters feed most actively at night (Hutchinson 1967) and we collected samples during the day, it is possible that diurnal differences in activity and respiration rates may have reduced in situ respiration rates relative to laboratory values. As Lampert (1984) indicated, potential factors affecting zooplankton respiration rates are not well known.

Nutrient regeneration rate data were more variable (through time and among ponds) than productivity and respiration rate data. Both $\mathrm{NH}_{3}-\mathrm{N}$ and $\mathrm{PO}_{4}-\mathrm{P}$ regeneration rates were within the ranges of values obtained by Korstad (1983). $\mathrm{NH}_{3}-\mathrm{N}$ regeneration rates were generally greater than $\mathrm{PO}_{4}-\mathrm{P}$ regeneration rates, again similar to the results of Korstad (1983). It is likely that measurable and reliable nutrient regeneration rates required both a "critical mass" of zooplankton in the incubated bottles and detectable background nutrient levels. If neither condition was met, as was probably the case for $\mathrm{PO}_{4}-\mathrm{P}$ on most sample dates, any nutrient regenerated during incubations would have been below detection limits by our analytical methods.

\section{Zooplankton community structure vs. function}

Community-level structure and function data were comparable in detecting variation among ponds: both were relatively insensitive to variation detected at finer scale. Schindler (1987) argued that structural data are more sensitive, and therefore better, early indicators of ecological stress than functional data. Likewise, our results call into question the ability of community-level function analyses (e.g., secondary production, respiration) to detect finer scale, structural changes in communities. However, we suggest that differences of scale affect the value of typically measured structure and function variables, rather than an intrinsic insensitivity of functional measures. Structure is often measured as density or biomass of selected species, whereas function has often been measured at the community level (e.g., primary productivity). Based on our results, the sensitivity of structure would be reduced if aggregated to scales equivalent to those commonly used for functional measures. Comparisons of structure and function at unmatched hierarchical scales may confound judgment of the relative sensitivity of structure and function to detect differences among communities.

The insensitivity of community function to details of community structure does not argue for the discontinuance of functional measurements in zooplankton community ecology. Rather, it argues for further scaleequivalent comparisons of structure and function. Community function offers an entirely different, potentially valuable perspective from community structure (O'Neill et al. 1986). We know of no other published studies evaluating structure and function of early 
zooplankton communities, nor of scale effects on relative sensitivities of structure and function. More work on structure-function comparisons and on other, early aquatic communities is needed for comparison to our results.

\section{Community assembly}

In effect, our study comprised a test of "null-set", expectations for all four species-area hypotheses (Williams 1943, MacArthur and Wilson 1967, Connell 1978, Coleman 1981). A logical extension of all four hypotheses to same-sized, closely spaced and environmentally similar ponds is that colonization should have occurred similarly among all ponds, regardless of the length of time involved and irrespective of concerns with "complete" colonization. The fact that colonization did not occur similarly in similar ponds indicates that factors other than those proposed by species-area hypotheses must be considered.

None of the species-area hypotheses consider interspecific variation in colonization rate (vagility). The Equilibrium Hypothesis (MacArthur and Wilson 1967) regards colonization as a process dependent on factors such as distance from propagule source and island size, but ignores interspecific differences in vagility. The Random Placement Hypothesis (Coleman et al. 1982) assumes that all organisms are unrestricted in their movement among sites, and is therefore of limited use for organisms with restricted vagility. Also, the Habitat Diversity (Williams 1943) and Intermediate Disturbance (Connell 1978) hypotheses make no explicit assumptions regarding the effects of differential vagility among colonizing organisms on resulting community composition.

Our results and the analyses of Jenkins (1995) indicate that zooplankton community composition was limited by dispersal, in addition to local processes affecting species' success upon arrival. Zooplankton have been supposed to disperse readily; dispersal must be all the more important to communities composed of organisms known to disperse poorly! It is not appropriate to state that zooplankton, in general, disperse readily; some do, but some do not. More detailed analyses of dispersal mechanisms are needed to understand why zooplankton vary in dispersal rates.

Dispersal processes and the resulting contingencies of colonization history have also been indicated as regulators of community composition in marine communities (Sale 1977, Sutherland and Carlson 1977, Underwood and Denley 1984, Roughgarden et al. 1987), forests (McCune and Allen 1985, Hubbell and Foster 1986), and prairie grasslands (Tilman 1997). The fact that dispersal has been found to be a regulator of community composition in several, widely different ecosystems suggests that supply-side concepts (e.g., Lewin 1986, Roughgarden et al. 1987) for community assembly may be a generality, and that greater attention should be given to regional-scale processes that reg- ulate local community structure (Ricklefs 1987). Metapopulation theory also focuses attention on dispersal among local populations, but some metapopulation concepts (e.g., rescue effect; Gotelli 1991) may not apply to species having limited dispersal. Instead, such species may exhibit little interaction among local populations, little gene flow, and persistent founder effects (Boileau and Hebert 1991, Hebert and Wilson 1994).

Organismal transport (dispersal, colonization, invasion) is a continuous process occurring in all ecosystems, whether young, old, disturbed, or pristine, and the details may be critical to the resulting communities that assemble. The successful result of dispersal (colonization) is easiest to study in new systems, but dispersal is a continuous process that does not cease and is the basis of metapopulation theory. Given that premise, questions of dispersal rates, variation among species, and processes affecting those rates become important to understanding patterns of community structure, and are discussed next.

Quorum effects.-Rapid dispersal among sites should promote the importance of local-scale regulators of community composition, analogous to general, prompt attendance to quickly form a quorum in a meeting. Regional-scale processes of arrival should be reduced in importance thereafter, unless late-arriving species are influential.

In situ studies of competition, predation, etc. implicitly assume a "quorum" has been reached, but may reveal little interspecific interaction if a community is not yet regulated by those processes (e.g., Underwood and Denley 1984). We think an explicit recognition of this commonly assumed "quorum" concept and examination of its validity is necessary to adequately evaluate the relative importance of regional- and localscale processes in community structure and function.

Limited dispersal should prolong the ascendancy of local-scale regulators of community composition (competition, predation, habitat availability, etc.), by delaying the presence of some species for participation in interspecific interactions and by accentuating the importance of species' arrivals in a relatively depauperate community. Therefore, generally low dispersal rates should increase the importance of dispersal as a regulator of community composition (Robinson and Edgemon 1988), while rapid dispersal of species to a site should reduce the importance of dispersal and promote the importance of local-scale regulatory processes (formation of a "quorum"). When the successional transition from regional to local regulation of community structure occurs is surely system-dependent, but should be of interest for understanding which processes are most important in regulating pattern.

This "quorum" concept is similar to MacArthur and Wilson's (1967) equilibrium species number, which is the balance between immigration and extinction rates. However, extinctions are not necessary to reach a "quorum," only that a shift between primacy of re- 
gional- and local-scale processes occurs. A "quorum" is therefore more subtle than an equilibrium species number, but may be detectable by two approaches: (1) experimental invasions of communities with varying structure, because biotic resistance to an invader (Simberloff 1986) may be greater with a "quorum"; and (2) experimental manipulations of competitor or predator populations in communities with varying structure, because multiple biotic interactions should stabilize community structure with a "quorum."

Colonization may be less important as a regulatory process in ecosystems that have accrued many species, but that argument may depend heavily on species-specific traits. The fact that exotic species disrupt ecosystems worldwide is testimony to the importance of some "late-arriving" species (Drake et al. 1989), and metapopulation dynamics are increasingly recognized as valuable concepts for local populations (e.g., Tilman 1997). For these reasons, a "quorum" may be difficult to demonstrate, and regional processes would be indicated as having continuing importance.

Priority effects. - Species do not disperse equally well, and vagile species are not necessarily poor competitors; in fact, it is difficult to predict which species will be strong invaders (Drake et al. 1989). Therefore, an influential species (e.g., dominant competitor) may sometimes colonize before other species. If so, that species may have lasting priority effects (Paine 1977) on later community composition.

Priority effects can occur at any time during community assembly; what is of interest is a species' effect on subsequent community composition. However, priority effects may be more likely in a simple community than a complex community: a dominant species (e.g., generalist predator) may regulate a simple community ("pre-quorum"), but could have a diluted influence, and itself be regulated by other species in a complex community ("post-quorum"). Therefore, dispersal processes may prescribe the importance of priority effects on community composition by regulating the availability of species to be affected. It is possible that tests for priority effects may be an additional way to detect the presence of a "quorum."

McCune and Allen (1985) concluded that historical factors, including colonization, had a lasting effect on old-growth forest composition. Their study was retrospective on old-growth forests, whereas our study was contemporary on zooplankton (organisms with brief life histories). We can only speculate on the extent that first-year colonization patterns would affect future zooplankton community composition in the experimental ponds. However, priority effects may have become important in the ponds, if species colonizing the ponds affected subsequent community composition. Others (e.g., Robinson and Dickerson 1987, Drake 1991, Alford and Wilbur 1985) have directly demonstrated the importance of priority effects in aquatic communities.

\section{CONCLusions}

Similar, new ponds developed different zooplankton communities due to different colonization histories. Therefore, colonization history may be an important regulator of other communities comprised of species expected to disperse less readily than zooplankton, up to and including old-growth forests (McCune and Allen 1985).

Our results indicate complex contingencies occured in both initiation and development of zooplankton assemblages, and do not support expectations derived from literature (zooplankton disperse readily) and from species-area hypotheses (similar places should have similar assemblages). That natural zooplankton communities are subject to contingencies is a platitude, but our formal understanding of zooplankton ecology has not traditionally considered this set of contingencies.

Colonization history's effects on the details of zooplankton community structure did not clearly translate to community-level structure and function. Community structure and function may be comparable in sensitivity for detecting change (Schindler 1987), but need to be compared at equivalent scales to better address the transfer of effects among and within the dual hierarchies of structure and function (O'Neill et al. 1986).

Finally, recognition of regional-scale processes that affect local-scale pattern (e.g., Lewin 1986, Robinson and Dickerson 1987, Roughgarden et al. 1987, Ricklefs 1987 ) is important for addressing decades-old questions about processes regulating communities. Dispersal rates predetermine the importance of local community regulators (e.g., competition, predation). A "quorum" of interacting species may form rapidly if dispersal is rapid, but slow dispersal rates may constrain development of locally-regulated communities, and may promote the importance of priority effects among the dispersal-limited community.

\section{ACKNOWLEDGMENTS}

We are grateful for the assistance provided by the following persons: Mike Rosenzweig, Keith Dockery, Dan Krause, Scott Smedley, Tim Morgan, and Bobbie Niederlehner. This project benefited from suggestions provided by Fred Benfield, George Simmons, Reese Voshell, and Jack Webster. We are also grateful to Eric Smith for statistical advice. This manuscript was greatly improved by the comments of Carol Folt, Kathy Cottingham, and James Drake. This research was supported in part by: Virginia Polytechnic Institute and State University; Sigma Xi, the Scientific Research Society; and the U.S. Environmental Protection Agency (Cooperative Agreements CR-814358-01-0 and 814358-02-1). This research does not necessarily reflect USEPA views, nor is an official endorsement implied.

\section{Literature Cited}

Ahmed, S. I., R. A. Kenner, and F. D. King. 1976. Preservation of enzymic activity in marine plankton by low-temperature freezing. Marine Chemistry 4:133-139.

Alford, R. A., and H. M. Wilbur. 1985. Priority effects in experimental pond communities: competition between Bufo and Rana. Ecology 66:1097-1105.

APHA, et al. 1985. Standard methods for the examination 
of water and wastewater. 16th edition. American Public Health Association, Washington, D.C., USA.

Arrhenius, O. 1921. Species and area. Journal of Ecology 9:95-99.

Austin, M. P. 1977. Use of ordination and other multivariate descriptive methods to study succession. Vegetatio 35:165175 .

Begon, M., J. L. Harper, and C. R. Townsend. 1990. Ecology: individuals, populations, and communities. Second edition. Blackwell, London, UK

Berg, D. J., and D. W. Garton. 1994. Genetic differentiation in North American and European populations of the cladoceran Bythotrephes. Limnology and Oceanography 39 : $1503-1516$.

Boileau, M. G., and P. D. N. Hebert. 1991. Genetic consequences of passive dispersal in pond-dwelling copepods Evolution 45:721-733

Bottrell, H. H., A. Duncan, Z. M. Gliwicz, E. Grygierek, A Herzig, A. Hillbricht-Ilkowska, H. Kurasawa, P. Larsson, and T. Weglenska. 1976. A review of some problems in zooplankton production studies. Norwegian Journal of Zoology 24:419-456.

Brown, J. H., and A. C. Gibson. 1983. Biogeography. C. V. Mosby, St. Louis, Missouri, USA.

Caley, M. J., and D. Schluter. 1997. The relationship between local and regional diversity. Ecology 78:70-80.

Carpenter, S., editor. 1988. Complex interactions in lake communities. Springer-Verlag, New York, New York, USA

Coleman, B. D. 1981. On random placement and speciesarea relations. Mathematical Biosciences 54:191-215.

Coleman, B. D., M. A. Mares, M. R. Willig, and Y.-H. Hsieh 1982. Randomness, area, and species richness. Ecology 63: $1121-1133$.

Connell, J. H. 1978. Diversity in tropical rain forests and coral reefs. Science 199:1302-1310.

Connell, J. H., and W. P. Sousa. 1983. On the evidence needed to judge ecological stability or persistence. American Naturalist 121:789-824.

Crossland, N. O., and C. J. M. Wolff. 1985. Fate and biological effects of pentachlorophenol in outdoor ponds. Environmental Toxicology and Chemistry 4:73-86.

deNoyelles, F., W. D. Kettle, and D. E. Sinn. 1982. The responses of plankton communities in experimental ponds to atrazine, the most heavily used pesticide in the United States. Ecology 63:1285-1293.

De Stasio, B. T., Jr. 1991. The seed bank of a freshwater crustacean: copepodology for the plant ecologist. Ecology 70:1377-1389.

Digby, P. G. N., and R. A. Kempton. 1987. Multivariate analysis of ecological communities. Chapman and Hall, London, UK

Doohan, M., and V. Rainbow. 1971. Determination of dry weights of small Aschelminthes. Oecologia 6:380-383.

Drake, J. A., H. A. Mooney, and F. di Castri et al., editors. 1989. Biological invasions. A global perspective. SCOPE 37. John Wiley and Sons, New York, New York, USA.

Drake, J. A. 1991. Community-assembly mechanics and the structure of an experimental species ensemble. American Naturalist 137:1-26.

Dumont, H. J., I. van de Velde, and S. Dumont. 1975. The dry weight estimate of biomass in a selection of Cladocera, Copepoda and Rotifera from the plankton, periphyton and benthos of continental waters. Oecologia 19:75-97.

Edmondson, W. T., and G. G. Winberg, editors. 1971. A manual on methods for the assessment of secondary productivity in fresh waters. IBP Handbook Number 17. Blackwell, Oxford, UK.

Giddings, J. M., P. J. Franco, R. M. Cushman, L. A. Hook, G. R. Southworth, and A. J. Stewart. 1984. Effects of chronic exposure to coal-derived oil on freshwater ecosys- tems, II. Experimental ponds. Environmental Toxicology and Chemistry 3:447-464.

Gotelli, N. J. 1991. Metapopulation models: the rescue effect, the propagule rain, and the core-satellite hypothesis. American Naturalist 138:768-776.

Hairston, N. G., Jr., T. A. Dillon, and B. T. De Stasio, Jr. 1990. A field test for the cues of diapause in a freshwater copepod. Ecology 71:2218-2223.

Hall, D. J., W. E. Cooper, and E. E. Werner. 1970. An experimental approach to the production dynamics and structure of freshwater animal communities. Limnology and Oceanography 15:839-928.

Haney, J. F., and D. J. Hall. 1973. Sugar-coated Daphnia: a preservation technique for Cladocera. Limnology and Oceanography 18:331-333.

Hebert, P. D. N., and C. C. Wilson. 1994. Provincialism in plankton: endemism and allopatric speciation in Australian Daphnia. Evolution 48:1333-1349.

Hubbell, S. P., and R. B. Foster. 1986. Biology, chance, and history and the structure of tropical rain forest communities. Pages 314-330 in J. Diamond and T. J. Case, editors. Community ecology. Harper and Row, New York, New York, USA

Hurlbert, S. H. 1984. Pseudoreplication and the design of ecological field experiments. Ecological Monographs 54: 187-211.

Hutchinson, G. E. 1967. A treatise on limnology. Volume II. Introduction to lake biology and the limnoplankton. John Wiley and Sons, New York, New York, USA.

Jenkins, D. G. 1990. Structure and function of zooplankton colonization in twelve new experimental ponds. Dissertation. Virginia Polytechnic Institute and State University, Blacksburg, Virginia, USA.

- 1995. Dispersal-limited zooplankton distribution and community composition in new ponds. Hydrobiologia 313/314:15-20.

Kerfoot, W. C., and A. Sih, editors. 1987. Predation: direct and indirect impacts on aquatic communities. University Press of New England, Hanover, New Hampshire, USA.

King, C. E. 1980. The genetic structure of zooplankton populations. Pages 315-328 in W. C. Kerfoot, editor. Evolution and ecology of zooplankton communities. University Press of New England, Hanover, New Hampshire, USA.

Kleinbaum, D. G., L. L. Kupper, and K. E. Muller. 1988. Applied regression analysis and other multivariate methods. Second edition. PWS-Kent, Boston, Massachusetts, USA.

Korstad, J. 1983. Nutrient regeneration by zooplankton in southern Lake Huron. Journal of Great Lakes Research 9: 374-388.

Lampert, W. 1984. The measurement of respiration. Pages 413-468 in J. A. Downing and F. H. Rigler, editors. A manual of methods for the assessment of secondary productivity in fresh waters. IBP Handbook Number. 17, Second edition. Blackwell, London, UK.

editor. 1985. Food limitation and the structure of zooplankton communities. Archive fur Hydrobiologie, Volume 21.

Lampert, W., and U. Sommer. 1997. Limnoecology. The ecology of lakes and streams. Oxford University Press, New York, New York, USA.

Layton, R. J., and J. R. Voshell, Jr. 1993. Colonization of new experimental ponds by benthic macroinvertebrates. Environmental Entomology 20:110-117.

Lewin, R. 1986. Supply-side ecology. Science 234:25-27.

Likens, G. E., and J. J. Gilbert. 1970. Notes on quantitative sampling of natural populations of planktonic rotifers. Limnology and Oceanography 15:816-820.

MacArthur, R. H., and E. O. Wilson. 1967. The theory of 
island biogeography. Princeton University Press, Princeton, New Jersey, USA.

MacIsaac, H. J, and J. J. Gilbert. 1991. Discrimination between exploitative and interference competition between Cladocera and Keratella cochlearis. Ecology 72:924-937.

Maguire, B., Jr. 1963. The passive dispersal of small aquatic organisms and their colonization of isolated bodies of water. Ecological Monographs 33:161-185.

Makarewicz, J. C., and G. E. Likens. 1979. Structure and function of the zooplankton community of Mirror Lake, New Hampshire. Ecological Monographs 49:109-127.

McCauley, E. 1984. The estimation of the abundance and biomass of zooplankton in samples. Pages 228-265 in J. A. Downing and F. H. Rigler, editors. A manual of methods for the assessment of secondary productivity in fresh waters. IBP Handbook Number 17. Second edition. Blackwell Scientific, London, UK.

McCune, B., and T. F. H. Allen. 1985. Will similar forests develop on similar sites? Canadian Journal of Botany 63: 367-376.

Morin, A., and P. Dumont. 1994. A simple model to estimate growth rate of lotic insect larvae and its value for estimating population and community production. Journal of the North American Benthological Society 13:357-367.

O'Neill, R. V., D. L. deAngelis, J. B. Waide, and T. F. H. Allen. 1986. A hierarchical concept of ecosystems. Princeton University Press, Princeton, New Jersey, USA.

Owens, T. G., and F. D. King. 1975. The measurement of respiratory electron-transport-system activity in marine zooplankton. Marine Biology 30:27-36.

Pace, M. L., and J. D. Orcutt, Jr. 1981. The relative importance of protozoans, rotifers, and crustaceans in a freshwater zooplankton community. Limnology and Oceanography 26:822-830.

Paine, R. T. 1977. Controlled manipulations in the marine intertidal zone, and their contributions to ecological theory. Special Publications of the Academy of Natural Sciences, Philadelphia 12:245-270.

Parma, S. 1969. The life cycle of Chaoborus crystallinus (de Geer) (Diptera, Chaoboridae) in a Dutch pond. International Vereinigung für theoretische und angewandte Limnologie, Verhandlungen 17:888-894.

Pennak, R. W. 1989. Fresh-water invertebrates of the United States. Third edition. John Wiley and Sons, New York, New York, USA.

Proctor, V. W. 1964. Viability of crustacean eggs recovered from ducks. Ecology 45:656-658.

Proctor, V. W., and C. R. Malone. 1965. Further evidence of the passive dispersal of small aquatic organsims via the intestinal tract of birds. Ecology 46:728-729.

Proctor, V. W., C. R. Malone, and V. L. DeVlaming. 1967. Dispersal of aquatic organisms: viability of disseminules recovered from the intestinal tract of captive killdeer. Ecology 48:672-676.

Ricklefs, R. E. 1987. Community diversity: relative roles of local and regional processes. Science 235:167-171.

Rigler, F. H., and J. A. Downing. 1984. The calculation of secondary productivity. Pages 19-58 in J. A. Downing and F. H. Rigler, editors. A manual of methods for the assessment of secondary productivity in fresh waters. IBP Hand- book Number 17. Second edition. Blackwell Scientific, London, UK.

Robinson, J. V., and J. E. Dickerson, Jr. 1987. Does invasion sequence affect community structure? Ecology 68:587595.

Robinson, J. V., and M. A. Edgemon. 1988. An experimental evaluation of the effect of invasion history on community structure. Ecology 69:1410-1417.

Rosenzweig, M. S., and A. L. Buikema, Jr. 1994. Phytoplankton colonization and seasonal succession in new experimental ponds. Environmental Toxicology and Chemistry 13:599-605.

Roughgarden, J., S. Gaines, and S. Pacala. 1987. Supplyside ecology: the role of physical transport processes. Pages 459-486 in P. Giller and J. Gee, editors. Organization of communities: past and present. Blackwell Scientific, London, UK.

Ruttner-Kolisko, A. 1977. Suggestions for biomass calculations of plankton rotifers. Archiv für Hydrobiologie Beiheft Ergebnisse der Limnologie 8:71-76.

Sale, P. 1977. Maintenance of high diversity in coral reef fish communities. American Naturalist 111:337-359.

SAS Institute. 1985. SAS user's guide: statistics. Version 5. SAS Institute, Cary, North Carolina, USA.

Schindler, D. W. 1987. Detecting ecosystem responses to anthropogenic stress. Canadian Journal of Fisheries and Aquatic Sciences 44(Supplement 1):6-25.

Simberloff, D. 1986. Introduced insects: a biogeographic and systematic perspective. Pages 3-26 in H. Mooney and J. Drake, editors. The ecology of biological invasions of North America and Hawaii. Springer-Verlag, New York, New York, USA.

Sokal, R. R., and F. J. Rohlf. 1981. Biometry. Second edition. W. H. Freeman, San Francisco, California, USA.

Sprules, W. G., H. P. Riessen, E. H. Jin. 1990. Dynamics of the Bythotrephes invasion of the St. Lawrence Great Lakes. Journal of Great Lakes Research 16:346-351.

Steedman, H. F. 1976. General and applied data on formaldehyde fixation and preservation of marine zooplankton. Pages 103-155 in H. F. Steedman, editor. Zooplankton fixation and preservation. UNESCO, Paris, France.

Sutherland, J. P., and R. H. Karlson. 1977. Development and stability of the fouling communities at Beaufort, N.C. Ecological Monographs 47:425-446.

Tarapchak, S. J., S. M. Bigelow, and C. Rubitschun. 1982. Soluble reactive phosphorus measurements in Lake Michigan: filtration artifacts. Journal of Great Lakes Research 8:550-557.

Tilman, D. 1997. Community invasibility, recruitment limitation, and grassland biodiversity. Ecology 78:81-92.

Underwood, A. J., and E. J. Denley. 1984. Paradigms, explanations and generalizations in models for the structure of intertidal communities on rocky shores. Pages 151-180 in D. R. Strong, Jr., D. Simberloff, L. G. Abele, and A. B. Thistle, editors. Ecological communities: conceptual issues and the evidence. Princeton University Press, Princeton, New Jersey, USA.

Wetzel, R. G. 1983. Limnology. Second edition. Saunders, Philadelphia, Pennsylvania, USA.

Williams, C. B. 1943. Area and number of species. Nature 152:264-267. 
APPENDIX

Environmental variables (monthly means \pm 1 standard deviation for all ponds).

\begin{tabular}{|c|c|c|c|c|c|c|}
\hline Month & $\begin{array}{c}\text { Temperature } \\
\left({ }^{\circ} \mathrm{C}\right)\end{array}$ & $\begin{array}{c}\text { Dissolved } \\
\text { oxygen } \\
(\mathrm{mg} / \mathrm{L})\end{array}$ & $\mathrm{pH}$ & $\begin{array}{l}\text { Hardness } \\
(\mathrm{mg} / \mathrm{L})\end{array}$ & $\begin{array}{l}\text { Alkalinity* } \\
\text { (mg/L) }\end{array}$ & $\begin{array}{c}\text { Conductivity* } \\
(\mu S)\end{array}$ \\
\hline Feb. 1988 & $6.68 \pm 0.11$ & $10.80 \pm 0.07$ & $6.80 \pm 0.09$ & $73.72 \pm 0.03$ & $30.31 \pm 0.04$ & $235.09 \pm 0.05$ \\
\hline Mar. & $8.27 \pm 0.04$ & $11.28 \pm 0.02$ & $6.85 \pm 0.08$ & $72.23 \pm 0.04$ & $31.88 \pm 0.07$ & $232.37 \pm 0.03$ \\
\hline Apr. & $14.48 \pm 0.09$ & $9.83 \pm 0.04$ & $7.11 \pm 0.09$ & $71.58 \pm 0.05$ & $33.58 \pm 0.08$ & $222.62 \pm 0.05$ \\
\hline May & $20.17 \pm 0.04$ & $8.82 \pm 0.10$ & $7.15 \pm 0.16$ & $73.83 \pm 0.06$ & $37.78 \pm 0.15$ & $211.75 \pm 0.06$ \\
\hline Jun. & $23.77 \pm 0.14$ & $7.99 \pm 0.12$ & $7.13 \pm 0.11$ & $72.18 \pm 0.06$ & $40.07 \pm 0.10$ & $210.71 \pm 0.07$ \\
\hline Jul. & $28.17 \pm 0.03$ & $7.76 \pm 0.08$ & $7.27 \pm 0.23$ & $73.56 \pm 0.07$ & $45.42 \pm 0.09$ & $212.00 \pm 0.08$ \\
\hline Aug. & $29.77 \pm 0.01$ & $7.41 \pm 0.08$ & $7.35 \pm 0.19$ & $69.49 \pm 0.13$ & $49.58 \pm 0.10$ & $195.94 \pm 0.11$ \\
\hline Sep. & $22.20 \pm 0.06$ & $9.04 \pm 0.08$ & $7.76 \pm 0.32$ & $53.82 \pm 0.28$ & $49.96 \pm 0.13$ & $194.62 \pm 0.10$ \\
\hline Oct. & $12.66 \pm 0.07$ & $10.24 \pm 0.03$ & $7.78 \pm 0.23$ & $41.45 \pm 0.15$ & $48.25 \pm 0.07$ & $185.12 \pm 0.09$ \\
\hline Nov. & $10.17 \pm 0.64$ & $9.35 \pm 0.62$ & $7.34 \pm 1.49$ & $31.91 \pm 1.04$ & $40.74 \pm 1.15$ & $144.10 \pm 1.78$ \\
\hline Dec. & $5.52 \pm 0.33$ & $11.81 \pm 0.03$ & $7.76 \pm 0.21$ & $40.02 \pm 0.37$ & $45.06 \pm 0.08$ & $170.94 \pm 0.17$ \\
\hline Jan. 1989 & $6.98 \pm 0.11$ & $11.77 \pm 0.02$ & $7.71 \pm 0.28$ & $54.30 \pm 0.11$ & $42.18 \pm 0.07$ & $167.32 \pm 0.11$ \\
\hline Feb. & $4.26 \pm 0.07$ & $11.85 \pm 0.03$ & $7.59 \pm 0.11$ & $59.72 \pm 0.10$ & $43.38 \pm 0.10$ & $168.08 \pm 0.09$ \\
\hline
\end{tabular}

* Denotes variables with significant difference among ponds $(P<0.05)$ by repeated-measures ANOVA.

APPENDIX. Extended.

\begin{tabular}{lcccccc}
\hline \hline \multicolumn{1}{c}{ Month } & $\begin{array}{c}\mathrm{NH}_{3}-\mathrm{N}^{*} \\
(\mathrm{mg} / \mathrm{L})\end{array}$ & $\begin{array}{c}\mathrm{NO}_{3}-\mathrm{N} \\
(\mathrm{mg} / \mathrm{L})\end{array}$ & $\begin{array}{c}\mathrm{NO}_{2}-\mathrm{N} \\
(\mathrm{mg} / \mathrm{L})\end{array}$ & $\begin{array}{c}\mathrm{PO}_{4}-\mathrm{P} \\
(\mathrm{mg} / \mathrm{L})\end{array}$ & $\begin{array}{c}\text { Secchi* } \\
(\mathrm{m})\end{array}$ & $\begin{array}{c}\mathrm{Chl} a^{*} \\
\left(\mathrm{mg} / \mathrm{m}^{3}\right)\end{array}$ \\
\hline Feb. 1988 & $0.02 \pm 0.02$ & $0.80 \pm 0.31$ & $0.05 \pm 0.05$ & $0.07 \pm 0.05$ & $0.83 \pm 0.06$ & $0.02 \pm 0.06$ \\
Mar. & $0.04 \pm 0.04$ & $1.17 \pm 0.16$ & $0.03 \pm 0.04$ & $0.01 \pm 0.01$ & $0.85 \pm 0.03$ & $0.18 \pm 0.31$ \\
Apr. & $0.05 \pm 0.05$ & $0.49 \pm 0.28$ & $0.20 \pm 0.20$ & $0.01 \pm 0.01$ & $0.94 \pm 0.06$ & $1.33 \pm 0.39$ \\
May & $0.07 \pm 0.04$ & $0.29 \pm 0.28$ & $0.51 \pm 0.11$ & $0.04 \pm 0.04$ & $0.68 \pm 0.09$ & $2.88 \pm 0.71$ \\
Jun. & $0.04 \pm 0.04$ & $0.06 \pm 0.08$ & $0.39 \pm 0.40$ & $0.01 \pm 0.01$ & $0.79 \pm 0.07$ & $3.19 \pm 0.42$ \\
Jul. & $0.01 \pm 0.01$ & $0.06 \pm 0.08$ & $0.00 \pm 0.02$ & $0.02 \pm 0.01$ & $1.43 \pm 0.07$ & $2.55 \pm 0.45$ \\
Aug. & $0.01 \pm 0.03$ & $0.05 \pm 0.10$ & $0.01 \pm 0.01$ & $0.01 \pm 0.01$ & $1.43 \pm 0.08$ & $1.25 \pm 0.87$ \\
Sep. & $0.03 \pm 0.02$ & $0.02 \pm 0.04$ & $0.00 \pm 0.00$ & $0.00 \pm 0.00$ & $1.67 \pm 0.08$ & $1.64 \pm 1.45$ \\
Oct. & $0.03 \pm 0.02$ & $0.01 \pm 0.02$ & $0.00 \pm 0.00$ & $0.00 \pm 0.00$ & $1.57 \pm 0.08$ & $1.93 \pm 0.79$ \\
Nov. & $0.02 \pm 0.02$ & $0.00 \pm 0.01$ & $0.00 \pm 0.00$ & $0.08 \pm 0.15$ & $1.00 \pm 0.16$ & $2.16 \pm 0.70$ \\
Dec. & $0.02 \pm 0.02$ & $0.01 \pm 0.01$ & $0.27 \pm 0.29$ & $0.00 \pm 0.00$ & $1.31 \pm 0.10$ & $1.94 \pm 0.58$ \\
Jan. 1989 & $0.01 \pm 0.01$ & $0.02 \pm 0.03$ & $0.10 \pm 0.13$ & $0.01 \pm 0.00$ & $1.32 \pm 0.08$ & $3.58 \pm 0.73$ \\
Feb. & $0.01 \pm 0.01$ & $0.01 \pm 0.01$ & $0.01 \pm 0.01$ & $0.02 \pm 0.01$ & $1.32 \pm 0.08$ & $3.86 \pm 0.67$ \\
\hline
\end{tabular}

